livraisons

d'Histoire

de l'Architecture

\section{Livraisons de l'histoire de l'architecture}

28 | 2014

Jean-Louis Pascal

\title{
Dans le sillage d'Henri Labrouste, premiers travaux à la Bibliothèque nationale
}

On the Henri Labrouste's posterity : first works at the National Library Auf den Spuren von Henri Labrouste: erste Arbeiten an der Bibliothèque Nationale

Anne Richard-Bazire

\section{CpenEdition}

Journals

Édition électronique

URL : http://journals.openedition.org/lha/393

DOI : $10.4000 /$ lha.393

ISSN : 1960-5994

Éditeur

Association Livraisons d'histoire de l'architecture - LHA

Édition imprimée

Date de publication : 10 décembre 2014

Pagination : 71-98

ISSN : 1627-4970

\section{Référence électronique}

Anne Richard-Bazire, « Dans le sillage d'Henri Labrouste, premiers travaux à la Bibliothèque nationale », Livraisons de l'histoire de l'architecture [En ligne], 28 | 2014, mis en ligne le 10 décembre 2015, consulté le 19 avril 2019. URL : http://journals.openedition.org/lha/393 ; DOl : 10.4000//ha.393

Ce document a été généré automatiquement le 19 avril 2019

Tous droits réservés à l'Association LHA 


\title{
Dans le sillage d'Henri Labrouste, premiers travaux à la Bibliothèque nationale
}

\author{
On the Henri Labrouste's posterity : first works at the National Library \\ Auf den Spuren von Henri Labrouste: erste Arbeiten an der Bibliothèque \\ Nationale
}

Anne Richard-Bazire

1 Le 6 juillet 1875, Jean-Louis Pascal est nommé architecte de la Bibliothèque nationale ${ }^{1}$, un an après la nomination de Léopold Delisle (1826-1910)² au poste d'administrateur général de la Bibliothèque. À l'annonce de sa nomination, il est abasourdi ; il vient tout juste d'apprendre qu'Henri Lefuel le « rétrograde » à l'agence des travaux du Louvre ; il écrit à Charles Garnier :

"C'était la place d'un gros bonnet et il était si naturel de vous donner la lourde succession d'un maître, que j'aurais été surpris que M. Delaborde eût eu une autre idée que de vous demander. De vous à moi que pourrait-il y avoir de commun ? [...] Mes remerciements à $\mathrm{M}$. de Cardaillac ${ }^{4}$ ont été très sincères, car si je tenais beaucoup à ne rien perdre de ce que je considère comme des droits acquis, mes prétentions ne s'étaient jamais élevées jusqu'à penser me trouver tout d'un coup sous le poids d'un héritage comme celui de Labrouste ${ }^{5}$."

2 Pascal met en place à la Bibliothèque une politique très différente de Labrouste qui n'avait pas hésité à détruire les bâtiments des siècles passés. Respectueux des œuvres de ses prédécesseurs, Pascal inaugure une attitude très nouvelle, celle du respect du patrimoine. Henri-Paul Nénot (1853-1934), son élève, ne s’y était pas trompé lorsqu'il écrivait: « «Pascal [...] fut un des premiers architectes qui respecta scrupuleusement l'œuvre des générations précédentes. Nous partageons tous aujourd'hui la manière de voir de mon maître et c'est cependant la première fois dans l'histoire de notre art que l'ensemble des artistes a le même scrupule ${ }^{6}$. » 


\section{Les tribulations de la Bibliothèque royale}

\section{Les origines de la Bibliothèque ${ }^{7}$}

3 Fondée par Charles V (1338-1380), dans la seconde moitié du XIVe siècle, la Librairie du roi est d'abord conservée au palais de la Cité puis au Louvre, aux trois derniers étages de la tour de la Fauconnerie, appelée de ce fait « tour de la Librairie ». Elle est située à l'angle N.O. du quadrilatère formé par les bâtiments du Louvre : « La tour de la Librairie fut par ordre du roi revêtue intérieurement de lambris de bois sculpté, les fenêtres furent garnies de grilles, des pupitres disposés autour de la pièce reçurent les volumes posés à plat, des sièges meublèrent cet intérieur; enfin une lampe d'argent fut suspendue à la voûte pour pouvoir y travailler la nuit. ${ }^{8}$. Déjà, "la consultation publique ou privée y [était] possible $»^{9}$. Cette tour disparut lors de la construction de la façade de l'horloge et on construisit à la place le pavillon central de cette façade.

Vendue en 1429 à Jean de Lancastre, duc de Bedford (1389-1435), régent du royaume pour le roi d'Angleterre, cette première bibliothèque est dispersée. On situe donc les vrais débuts de la Bibliothèque du roi au temps de Louis XI (1423-1483) dont les collections ne sont pas dispersées après la mort. Avec Charles VIII (1470-1498) et Louis XII (1462-1515), qui transportent les collections respectivement à Amboise et à Blois, la Bibliothèque royale s'accroît de manuscrits prélevés en Italie, de collections flamandes acquises par Louis XII et des volumes de la bibliothèque de son père, le poète Charles d'Orléans (1394-1465). François $1^{\text {er }}$ (1494-1547) et Guillaume Budé (1467-1540), «maître de la Librairie du roi ", vont, grâce au réseau des ambassadeurs et des voyageurs, acquérir manuscrits grecs, arabes, hébreux, pour la Bibliothèque. En 1537, François $1^{\mathrm{er}}$ instaure la première obligation du dépôt légal de tout nouveau livre imprimé et en 1544, lors du transport de la collection royale de Blois à Fontainebleau, elle compte déjà quatre mille cinq cents livres ${ }^{10}$. Henri II (1519-1559) augmente cette collection de celle que Catherine de Médicis (1519-1589) avait apportée de Florence et Charles IX (1550-1574) contribue à son développement en faisant de nombreuses acquisitions et en créant la fonction de " garde des Médailles ». Charles IX ramène la Bibliothèque royale à Paris dans un local du quartier latin à partir de 1567. Henri IV (1553-1610) la placera en 1594 dans les bâtiments du collège de Clermont ${ }^{11}$, rue Saint-Jacques, laissé vacant par les Jésuites expulsés de France.

5 En 1603, au retour des Jésuites, la Bibliothèque est transférée au couvent des Cordeliers ${ }^{12}$, dans la salle du cloître. À partir de 1622, pendant le règne de Louis XIII (1601-1643) et les vingt premières années de celui de Louis XIV, elle est conservée dans une maison appartenant à cet ordre, rue de la Harpe, près de l'église Saint-Côme.

En 1666, Colbert (1619-1683) qui l'avait considérablement enrichie - elle est alors la plus importante d'Europe - lui consacre « les maisons au bout de ses jardins $»^{13}$ (ill. 1) : il la fait transférer dans deux immeubles achetés par lui, tout à côté de son hôtel, rue Vivienne : « Les livres royaux étaient empilés, tant bien que mal, dans les logis vermoulus de l'hôtel Colbert, rue Vivienne, et le dépôt n'en était pas public [...] Les soixante-quinze mille volumes dont la collection se composait, occupaient vingt-cinq pièces poudreuses, mal éclairées, sans feu pendant l'hiver $»^{14}$. 


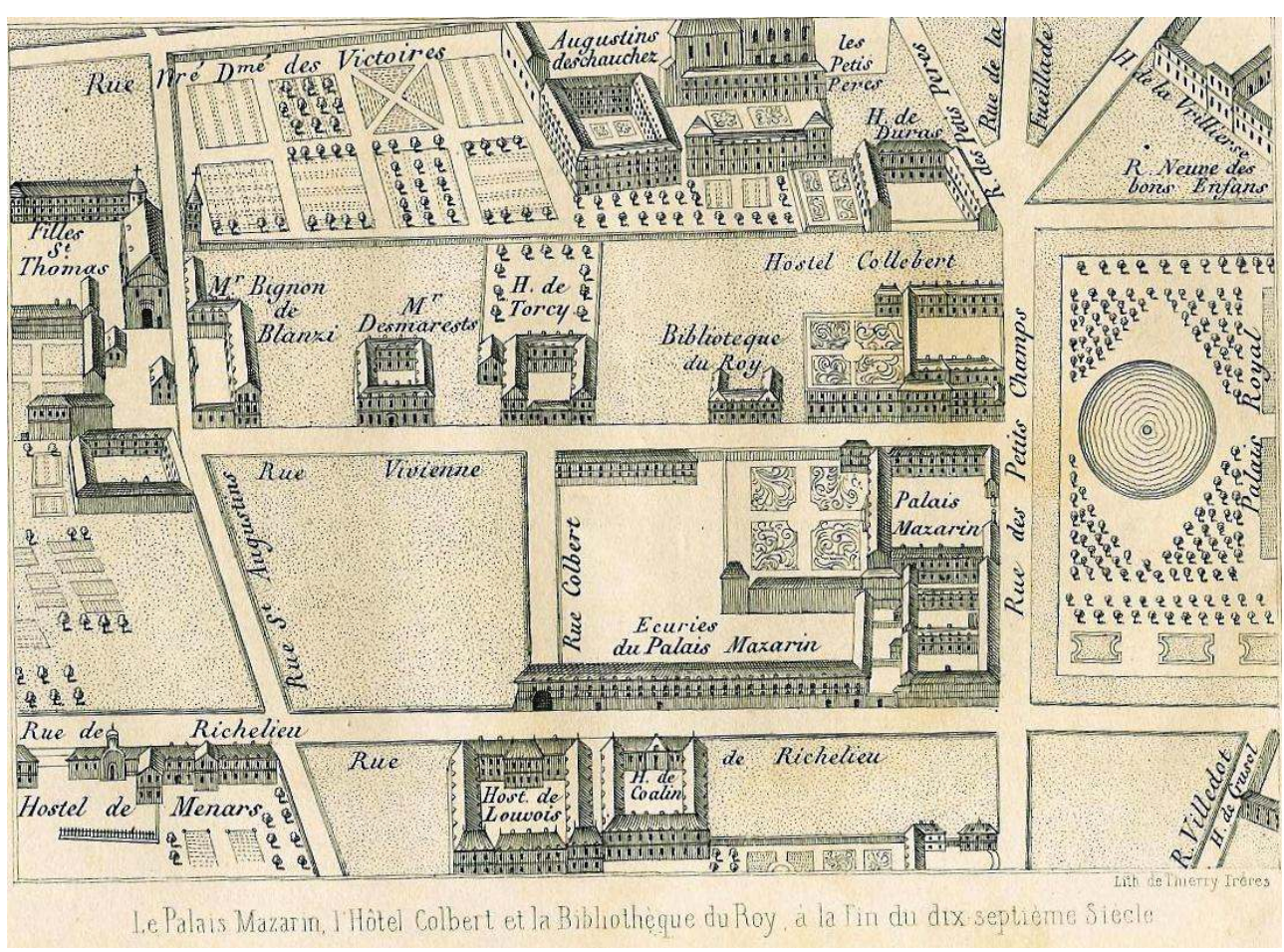

Architectes Pierre le Muet et François Mansart, entre la rue de Richelieu (à l'ouest), la rue Colbert (au nord), la rue Vivienne (à l'est) et la rue des-Petits-Champs (au sud), à la fin du XVIIe siècle

Léon de Laborde, De l'organisation des bibliothèques dans Paris, $4^{e}$ lettre : Le palais Mazarin et les habitations de ville et de campagne au XVII' siècle, Paris, A. Franck, 1845, p. 4.

7 Un premier projet de Jules Hardouin-Mansart (1646-1708) daté de 1685 plaçait la bibliothèque royale derrière la nouvelle place Louis-le-Grand (actuelle place Vendôme); un début de construction interrompue par la mort du marquis de Louvois (1639-1691) et une seconde conception de la place aboutirent à la destruction des bâtiments déjà élevés en 1694. Le 11 novembre 1716, le Régent (1674-1723) approuva la proposition de l'abbé de Louvois (1674-1719), alors garde de la Bibliothèque, de s'installer dans l'hôtel Tubeuf et la double galerie de Mansart et dans le quadrilatère bâti par Pierre Le Muet (1591-1669) sur la rue des Petits-Champs et la rue de Richelieu, héritage du duc de Nevers, mais la Compagnie des Indes et la banque de Law (1671-1729) s'emparant des locaux, le projet fut abandonné. En 1720, on abandonne un second projet d'installation de la Bibliothèque au Louvre, dans l'ancien appartement ${ }^{15}$ de Marie de Médicis (1573-1642), à cause de l'arrivée de l'Infante Marie-Anne-Victoire d'Espagne ${ }^{16}$.

\section{Le quadrilatère Richelieu aux XVII et XVIII ${ }^{e}$ siècles}

En 1720, la faillite de Law met à la disposition de la Bibliothèque ces bâtiments spacieux auxquels on avait déjà songé en 1716, juste en face de la propriété de Colbert, de l'autre côté de la rue Vivienne. Ces bâtiments constituent l'ancien palais de Mazarin (1602-1661), ensemble de constructions disparates, dont « la construction s'était étalée dans le temps sur plus d'un siècle et dans l'espace sur plus de quatorze mille mètres carrés ${ }^{17}$, bâtiments loués puis acquis par le cardinal Mazarin. 
La partie la plus ancienne en est l'hôtel dit «Tubeuf» (ill. 2), bâti en 1635 par l'entrepreneur Jean Thiriot d'après les dessins de l'architecte Pierre Le Muet à l'angle des rues Vivienne et Neuve-des-Petits-Champs pour Charles Duret de Chevry contrôleur général des finances, et acheté en 1641 à son fils par Jacques Tubeuf, influent intendant des finances du royaume et proche du cardinal Mazarin à qui il loue cet hôtel dès $1643^{18}$ (Mazarin l'achètera le 30 octobre $1649^{19}$ ).

\section{2 : L'Hôtel Tubeuf en 1858}

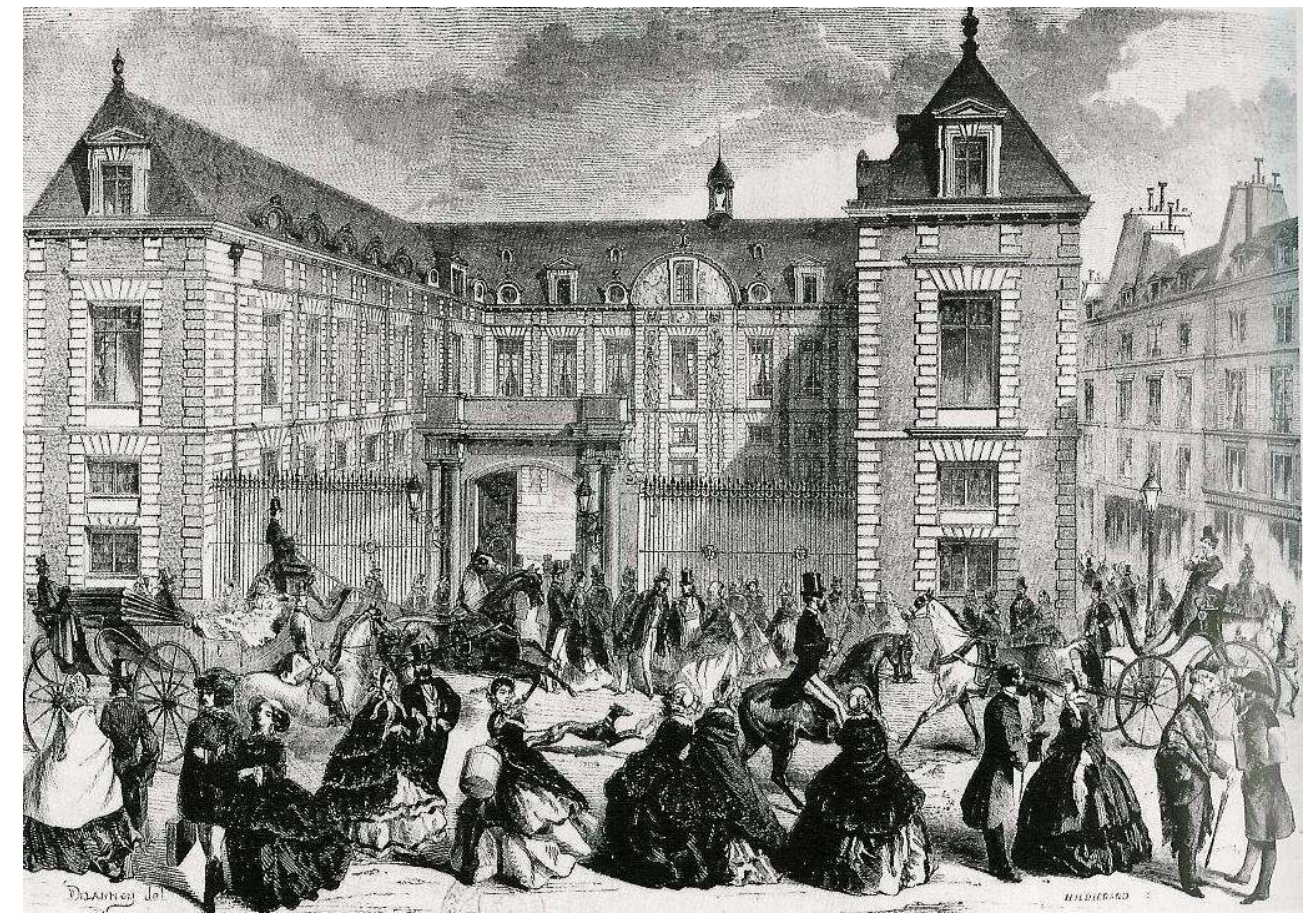

Pierre le Muet (architecte) et Jean Thiriot (entrepreneur)

Dominique Varry (dir.), Histoire des bibliothèques françaises. Les bibliothèques de la Révolution et du XIXe siècle : 1789-1914, Paris, éd. du Cercle de la Librairie, 1991, p. 338.

10 C'est cet hôtel en « U » que l'on voit encore aujourd'hui, les seules différences entre l'état primitif et l'état actuel étant la longueur des ailes, prolongées par la suite par François Mansart (1598-1666) jusqu'à la rue des Petits-Champs et la grille actuelle qui a remplacé une ancienne porte cochère encadrée par deux écuries en bordure de rue ${ }^{20}$.

Pour se loger, Jacques Tubeuf achète alors la pièce de terre attenante à l'hôtel Tubeuf et y fait construire par Pierre Le Muet trois corps de logis, connus sous le nom d'hôtel Duret de Chevry. En 1645, la galerie Mazarine est construite perpendiculairement à l'hôtel Tubeuf par François Mansart pour abriter les collections du Cardinal ${ }^{21}$. Celui-ci fait aussi construire entre 1646 et 1650 des appartements voûtés « dans le goût italien » sur la rue de Richelieu, à la suite de l'hôtel Duret de Chevry et les fait relier à la galerie Mazarine par un bâtiment dit « de la traverse ». Cette grande galerie longeant la rue de Richelieu détruite au XIX ${ }^{e}$ siècle par Henri Labrouste - formait, avec les jardins en retour sur la rue Vivienne, la partie de l'hôtel Mazarin qui revint à la mort de ce dernier, en 1661, à son neveu, Philippe Mancini, duc de Nevers (1641-1707)22. Elle abritait au rez-de-chaussée les écuries $^{23} \mathrm{du}$ Cardinal et au premier étage une galerie de peinture, une chapelle et la galerie de la bibliothèque ${ }^{24}$. 
«La porte d'entrée, située à peu près à la hauteur du milieu de la grande salle actuelle de travail, présentait un cintre surélevé, les montants en pierres de taille saillantes se terminant par des consoles qui portaient de chaque côté un trophée de boucliers et d'armures couronnés d'un casque à panache. Le reste de la façade, rue de Richelieu, était nu, sans ornement: un grand mur dont toutes les fenêtres étaient aveugles. En entrant par la grande porte de la rue de Richelieu, on se trouvait dans une première cour environnée de constructions assez simples, mais non dépourvues de caractère, les fenêtres s'ouvrant dans des arcades et reposant sur des balustres. Cette cour, dite basse-cour ou cour des écuries, se continuait à gauche, en suivant les galeries de la rue de Richelieu, par une seconde cour transformée en jardin. Les façades de Mansart se développaient ici avec plus d'intérêt que du côté de la rue. Le rez-de-chaussée offrait vingt-deux hautes fenêtres à amortissement semi-circulaire que décorait comme sculpture le faisceau de licteurs de Mazarin. Le $1^{\mathrm{er}}$ étage présentait trente fenêtres hautes, terminées par des linteaux que surmontaient, de deux l'une, des frontons triangulaires montés sur

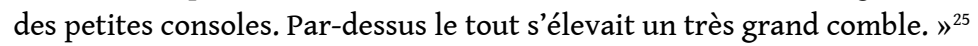

Le départ de la bibliothèque de Mazarin qui avait été, selon ses volontés, léguée au collège des Quatre-Nations, laissa vide une partie de cette grande galerie; le duc de Nevers n'en ayant jamais fait usage, céda en 1698, à titre de viager, à Anne-Thérèse de Marguenat de Courcelles, marquise de Lambert, l'extrémité nord de son hôtel ${ }^{26}$ qui avait une sortie particulière et pouvait être facilement disposée en une demeure élégante et commode. Cette dernière dépensa plus de cent mille francs pour disposer convenablement ce corps de bâtiment et pour construire, sur la nouvelle rue Mazarin, future rue Colbert, les communs $^{27}$ et la nouvelle entrée ${ }^{28}$ (ill.3).

\section{3 : Plan de la Bibliothèque nationale en 1850 avant les transformations d'Henri Labrouste}

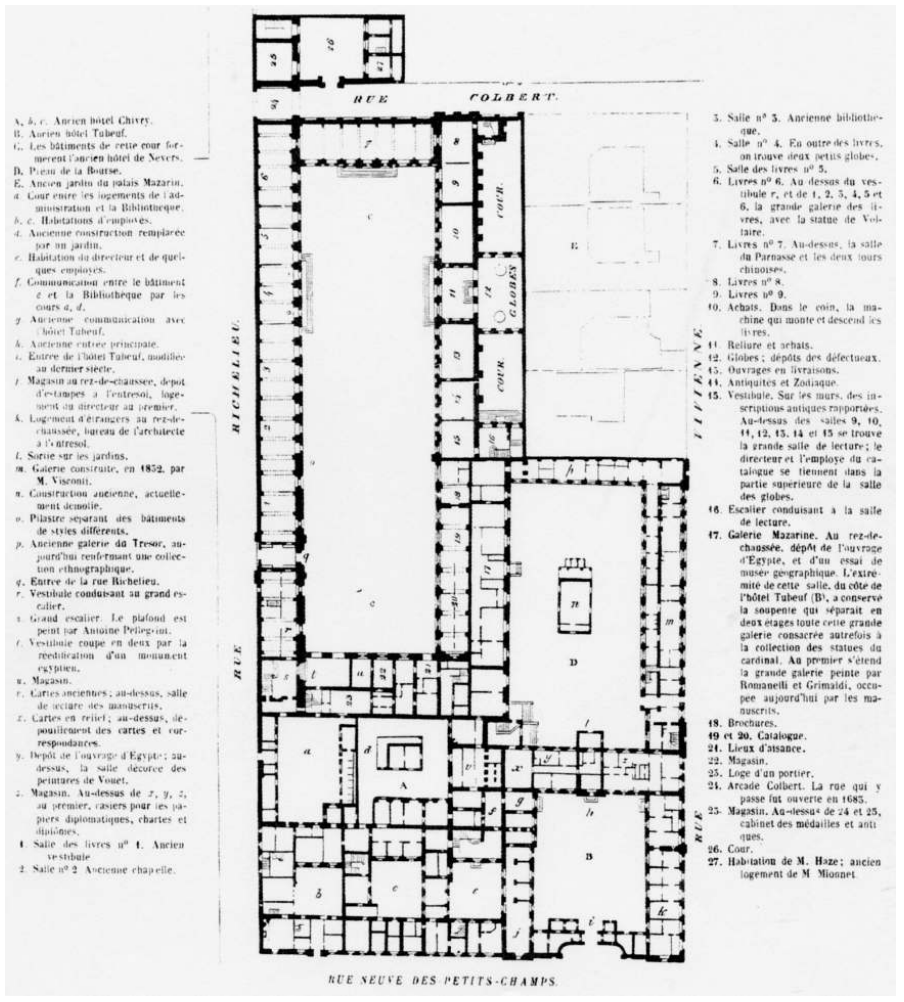

Léon de Laborde, De l'organisation des bibliothèques dans Paris, $8^{e}$ lettre : Étude sur la construction des bibliothèques dans Paris, Paris, A. Franck, 1845, pl. III, p. 14. 
effet, le marquis de Meilleraye, second héritier du Cardinal (car époux de sa nièce Hortense), qui lui avait légué l'hôtel Tubeuf, avait vendu à Colbert son jardin. Celui-ci y avait fait construire, le long de la rue Vivienne, des maisons de rapport, et pour en accroitre la valeur, on avait fait percer en 1683 une rue les faisant communiquer avec la rue de Richelieu; pour cela, on avait demandé au duc de Nevers le droit de percer une arcade (ill. 4) sous l'extrémité de son hôtel et en guise de remerciement on avait appelé la rue « Mazarin $»^{29}$.

\section{4 : L'arcade Colbert. Bibliothèque royale. 1646-1650}

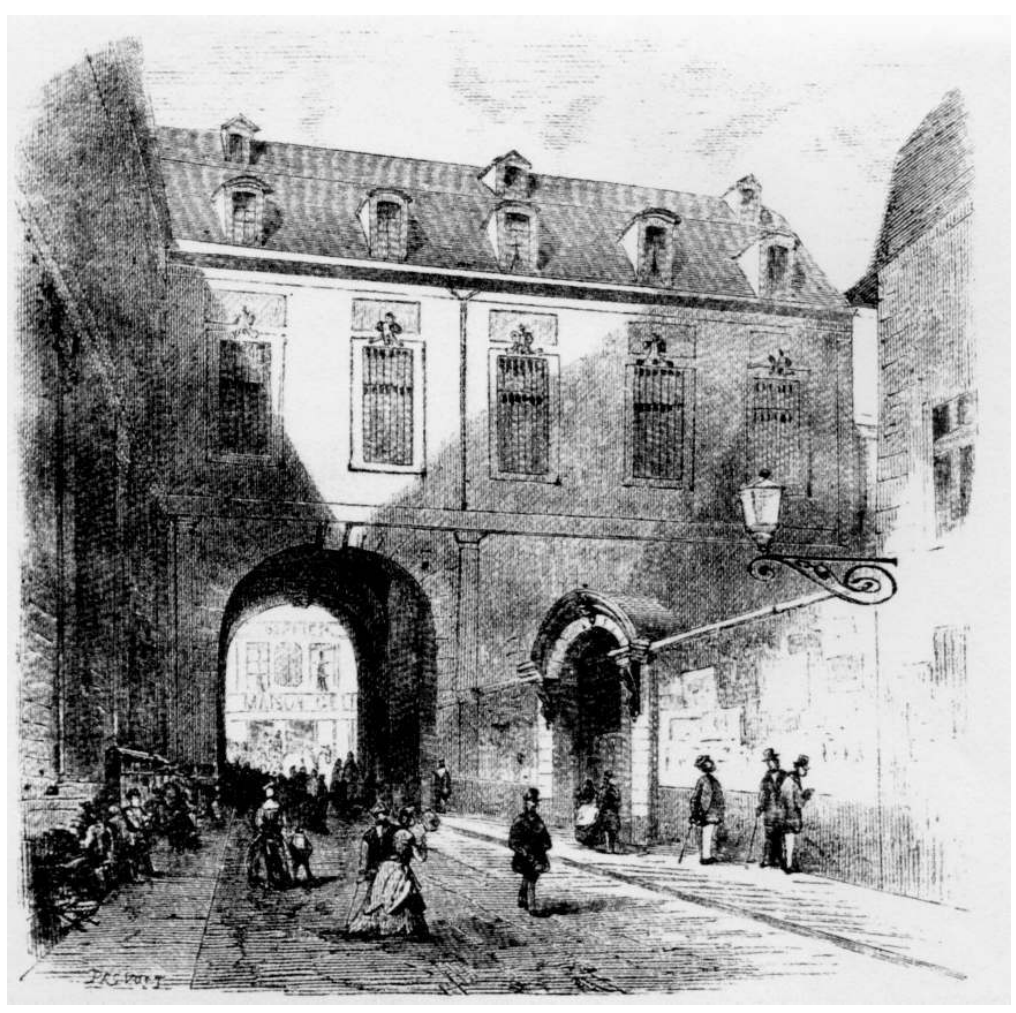

La rue Colbert ouverte en 1683 rejoint la rue de Richelieu au-delà de l'arcade

Dominique Varry (dir.), Histoire des bibliothèques françaises. Les bibliothèques de la Révolution et du XIXe siècle : 1789-1914, Paris, éd. du Cercle de la Librairie, 1991, p. 339

L'hôtel de Lambert abrita, de 1710 à 1733, un célèbre salon où se retrouvaient entre autres, Montesquieu et Marivaux ${ }^{30}$.

En 1719, lorsque John Law de Lauriston achète aux héritiers du Cardinal la totalité du palais Mazarin et à ceux de Colbert les cinq maisons construites le long de la rue Vivienne, il distribue les bureaux de la Compagnie des Indes et ses grands magasins dans le palais du duc de Mazarin, sur la rue Neuve-des-Petits-Champs; les comptoirs, les bureaux, l'imprimerie de la banque, l'affinage des métaux, les ateliers de la monnaie sont répartis dans l'ancien hôtel de Nevers. Les salons de réception occupent les anciens appartements de la duchesse de Nevers (situés dans la traverse) et une partie de la galerie sur la rue de Richelieu. On décide de plus de prolonger la galerie Mazarine jusqu'à la rue Colbert, et en abattant les maisons construites sur la rue Vivienne, on ouvre une place entourée d'arcades destinée à servir de bourse. L'architecte Claude Armand Mollet (1660-1742) fut l'auteur de cette transformation du palais Mazarin ${ }^{31}$. 


\section{La Bibliothèque s'installe rue de Richelieu}

16 La faillite de Law entraîne la confiscation des hôtels par le Régent Philippe d'Orléans (1674-1723) et laisse sans emploi les dépendances du palais parallèles à la rue de Richelieu. En 1721, le Régent décide d'y installer la Bibliothèque. L'abbé Bignon (1662-1743), successeur de l'abbé de Louvois comme garde de la Bibliothèque, obtient par arrêt du Conseil du 14 septembre 1721 l'affectation de la partie de l'hôtel de Nevers qui n'était pas occupée par la Compagnie des Indes, à la Bibliothèque encore entassée rue Vivienne $^{32}$. Le déménagement de la Bibliothèque commence le 25 septembre 1721, se déroule en trois fois, et s'étale sur deux ans, de 1721 à 1723 ; il est achevé le 16 mai $1724^{33}$. L'architecte Robert de Cotte (1656-1735) est chargé d'adapter les lieux à leur nouvelle destination, notamment la longue galerie, anciennement appelée « Neuve » située dans le prolongement de celle de Mansart ; c'est le bâtiment actuel du fond de la cour d'honneur, face à l'entrée de la Bibliothèque ${ }^{34}$. Robert de Cotte soigne particulièrement la menuiserie des meubles et des corps de bibliothèque destinés à toutes les salles où devaient être conservés les ouvrages. Cet aménagement avait donné lieu à de longues conversations entre l'architecte et le gardien-chef des livres du roi, l'abbé Bignon, qui tenait beaucoup à ne pas mettre de livres entre les croisées des fenêtres.

Robert de Cotte conserva les devis de ces travaux d'art, surveillés avec un soin jaloux et dont il avait fourni tous les croquis, tous les profils, depuis ceux des pilastres et des consoles, jusqu'aux moulures des meneaux pour les fenêtres, aux arêtes des dormants: «Ces ouvrages consistent en armoires pour placer les livres, dans les cinq pièces du grand étage, compris la galerie, dans les corps de logis de la rue de Richelieu, contenant ensemble soixante-dix-huit toises courantes sur vingt pieds et demi de haut environ ${ }^{35}$. Les travaux furent confiés à l'entrepreneur Leschaudelle, moyennant le prix de six cents livres par toise courante de haut en bas.

18 Les corps de bibliothèque régnaient dans toute la longueur des pièces, confectionnés en chêne et pourvus de distance en distance de pilastres agrémentés de sculptures en relief, avec, à leur sommet, des consoles enroulées, destinées à supporter le balcon. Au long de ce balcon, une rampe en fer forgé décorée $d^{\prime}$ " $X$ » en volutes reliées entre elles. On accédait à ce balcon par des escaliers engagés dans la muraille. Un corps de bibliothèque était fait ainsi : un socle de six pouces, cinq rangs d'in-folio de vingt-deux pouces, trois rangs d'in-quarto de treize pouces, le dessus du balcon ou socle de six pouces, trois rangs d'in-octavo et in-douze de dix pouces chacun et quatre rangs d'autres in-douze et in-seize de sept pouces chacun. Les consoles du haut des pilastres avaient surtout préoccupé Robert de Cotte. Elles devaient avoir deux pieds de saillie pour soutenir le balcon, être ornées de rouleaux en haut et en bas, avec des feuilles de refente galbées jusqu'aux caulicoles et des ornements tant sur le devant que sur les côtés, enroulements de palmettes et chutes de festons. La corniche en saillie du balcon avait la doucine décorée de feuilles de refente et, entre les feuilles, de culots et de graines, le tout de deux pouces et demi de circonférence. De Cotte veilla également à la corniche de plâtre à placer audessus des corps de bibliothèque, avec une frise en gorge de vingt pouces de pourtour, ornée de cartels et de trophées d'art dans la rencontre avec les pilastres des armoires ${ }^{36}$.

Les saisies révolutionnaires vont gonfler les collections de la Bibliothèque dont seulement deux parties étaient conçues pour abriter les collections : l'ancienne bibliothèque du Cardinal le long de la rue de Richelieu et les bâtiments qui ferment la cour d'honneur à 
l'est, achevés par Robert de Cotte, et au nord, le long de la rue Colbert, aile ${ }^{37}$ édifiée entre 1740 et $1749^{38}$. L'ancien hôtel Duret de Chevry avait été acquis en 1750 pour loger le personnel, et le petit hôtel Colbert, de l'autre côté de l'arcade du même nom ${ }^{39}$, abritait les médailles et le conservateur.

Ce n'est que dans les années 1820 que le palais Mazarin proprement dit reçoit la même destination que l'hôtel de Nevers: en 1825, la Bourse libère la galerie Mansart en s'installant au palais Brongniart; en octobre 1833, l'hôtel Tubeuf, libéré par les Finances depuis $1826^{40}$, est divisé en logements pour le personnel, puis on y installe le département des cartes et plans ${ }^{41}$. On doit à Louis Tullius Joachim Visconti (1791-1853), architecte de la Bibliothèque de 1828 à 1853, la création, en 1833, d'une salle publique de lecture au premier étage de l'aile est de la cour d'honneur ${ }^{42}$, l'installation du chauffage en 1839-1840 et la construction d'une nouvelle galerie parallèle à la galerie Mazarine, longeant la rue Vivienne. Commencée en 1831, à l'emplacement du préau de la Bourse, elle est achevée en $1847^{43}$.

\section{Henri Labrouste, architecte de la Bibliothèque}

Pendant toute la première moitié du XIXe siècle, de multiples projets de déménagement de la Bibliothèque sont envisagés ${ }^{44}$, mais après la mort de Visconti, on décide d'adapter les lieux déjà existants. En 1858, une commission est nommée pour réorganiser les collections et les services. Elle établit comme une nécessité future, l'acquisition des terrains occupés alors par des immeubles, à l'angle des rues Vivienne et Colbert. En attendant, on demande à Henri Labrouste un agrandissement des constructions au moyen d'une combinaison nouvelle du plan général, sur la même surface. Auréolé par la construction entre 1838 et 1850 de la bibliothèque Sainte-Geneviève ${ }^{45}$, il est nommé le 16 février 1854, architecte de la Bibliothèque impériale. Il détruit sur la rue Vivienne la galerie de Visconti constituée de portiques en pierre surmontés d'un toit mansardé sous lesquels étaient installées des boutiques, la remplace par une haute grille sur un mur bahut de pierre et réaménage le jardin en créant le bassin central avec quatre parterres. Pascal s'interroge sur l'origine de cette grille ${ }^{46}$ :

«Quand j'étais petit j'allais souvent jouer sur la place royale et un bon oncle - qui était du bâtiment - et qui m'initiait avec mélancolie à la décadence des hôtels intéressants du Temple et du Marais m'a plusieurs fois parlé de la belle grille à laquelle on avait substitué le modèle banal qui entoure le quinconce. À la même époque, en parisien que j'étais, et que je n'ai pas cessé d'être, je connaissais bien les boutiques [...] de marchands d'oiseaux, de vendeurs de vieilles gravures qu'abritait le bâtiment à arcades analogue à celui ${ }^{47}$ que je vais prochainement démolir sur le bâtiment en retour sur le jardin de la Bibliothèque rue Vivienne. La grille qui l'a remplacé avec un assez large reculement n'a dû être installée qu'en mars 1858 [...] De 1839 à 1858 il y a trop de marge pour qu'on ait pensé à doter la Bibliothèque à si longue échéance, et, d'ailleurs, on a à mon agence des restes d'études de la nouvelle grille que Labrouste a composée. Il se pourrait [...] qu'il se fût inspiré du souvenir de l'autre grille puisqu'à ce moment il faisait du Louis XIII dans l'hôtel de l'administrateur de la rue des Petits-Champs ${ }^{48}$, au point d'avoir considérablement mis du sien dans ces façades brique et pierre dont j'ai un souvenir bien misérable avant son intervention. C'est à la même époque qu'il a fait les deux hôtels pour les frères Fould, de même style, rue de Berry. Mais j'ai fait comparer avec les vues d'ailleurs assez fantaisistes et diverses - de la place Royale qu'on a au département des estampes, et on y trouve aucune analogie avec le motif actuel de la haute grille de la rue Vivienne. ${ }^{49}$ 
Henri Labrouste réhabilite l'hôtel Tubeuf et la galerie Mazarine où il installe au rez-dechaussée le département des estampes. La galerie Mazarine est surplombée d'une voûte surbaissée à compartiments de stuc dorés par Ottaviano Ottoviani ${ }^{50}$ peinte vers 1646-1647 par Giovanni Francesco Romanelli (1617-1662) ${ }^{51}$ et son atelier, dont Paolo Gismondi ; médaillons en camaïeu (ill. 5) et fresques illustrent des sujets mythologiques inspirés des Métamorphoses d'Ovide ${ }^{52}$.

\section{5 : Médaillon en camaïeu. Neptune}

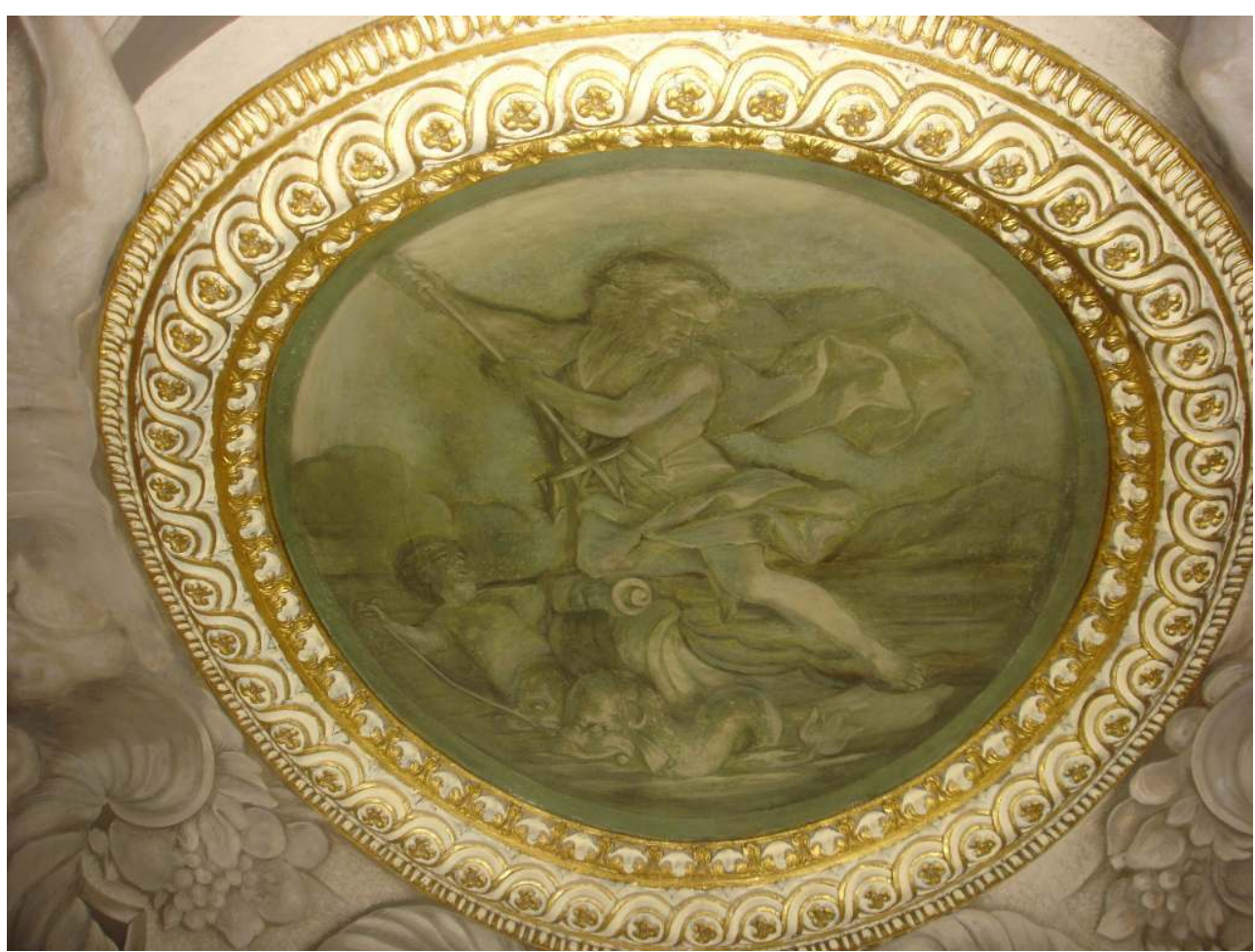

Galerie Mazarine. Bibliothèque nationale. 1646-1647. Compartiment de stuc doré par Ottaviano Ottoviani et peint par Giovanni Francesco Romanelli et son atelier dont Paolo Gismondi

Cl. auteur

Chacune des baies qui l'éclairent, surmontées d'une énorme coquille dorée (ill. 6) s'ouvre dans une embrasure peinte à fresque par l'artiste italien Grimaldi Bolognèse (1606-1680) ${ }^{53}$ 

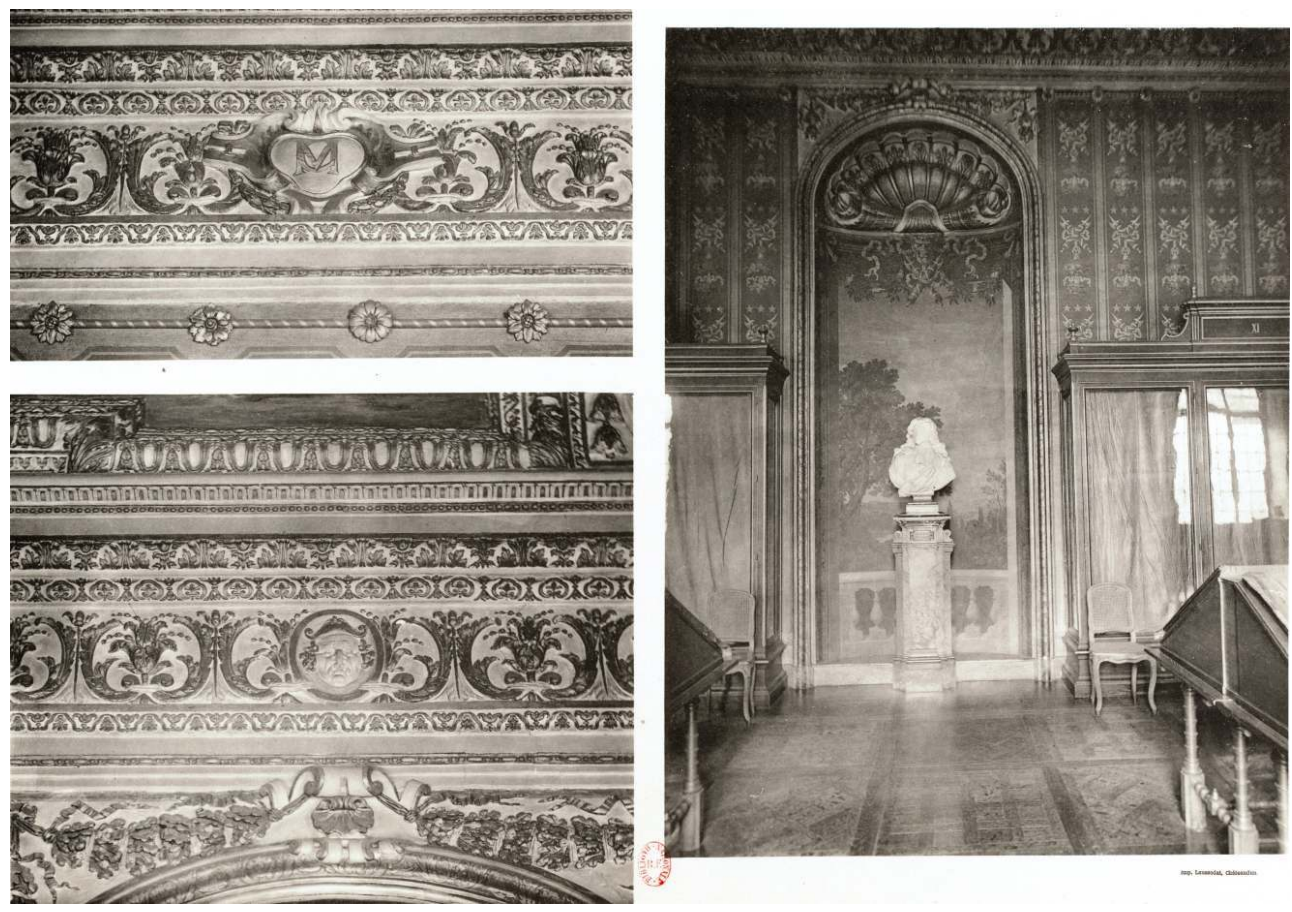

Architecte François Mansart. 1645. Peintures de Giovanni Francesco Romanelli et Grimaldi. Niche et détails du plafond

Arch. adm. B.n.F., 2007/061/001, pl. 110 à 112

«Les espaces entre les niches peintes par Grimaldi étaient autrefois tapissées de damas rouge cramoisi, semé des armes et du chiffre du Cardinal et rehaussé de passement d'or de Milan $\aleph^{54}$. En face, ces baies sont répétées par des baies exactement semblables, où des glaces tiennent lieu de fenêtres; en 1860, ces dernières avaient été condamnées depuis longtemps par des casiers de livres ${ }^{55}$ et la galerie était lézardée en maints endroits.

Des modifications faites à la charpente de la galerie à la demande de Romanelli - au départ prévue avec trois coupoles remplacées par une voûte continue en berceau expliquaient, selon Labrouste, une construction négligée à l'origine et son mauvais état de conservation ${ }^{56}$. Labrouste remplaça la charpente en bois par une charpente en fer et la suréleva pour ne pas détruire la voûte et pour masquer une différence des deux axes de $17 \% \%^{57}$. Il ajouta à l'extérieur la fausse balustrade en terre cuite au-dessus de la corniche, pour masquer l'élévation du comble (ill. 7). 


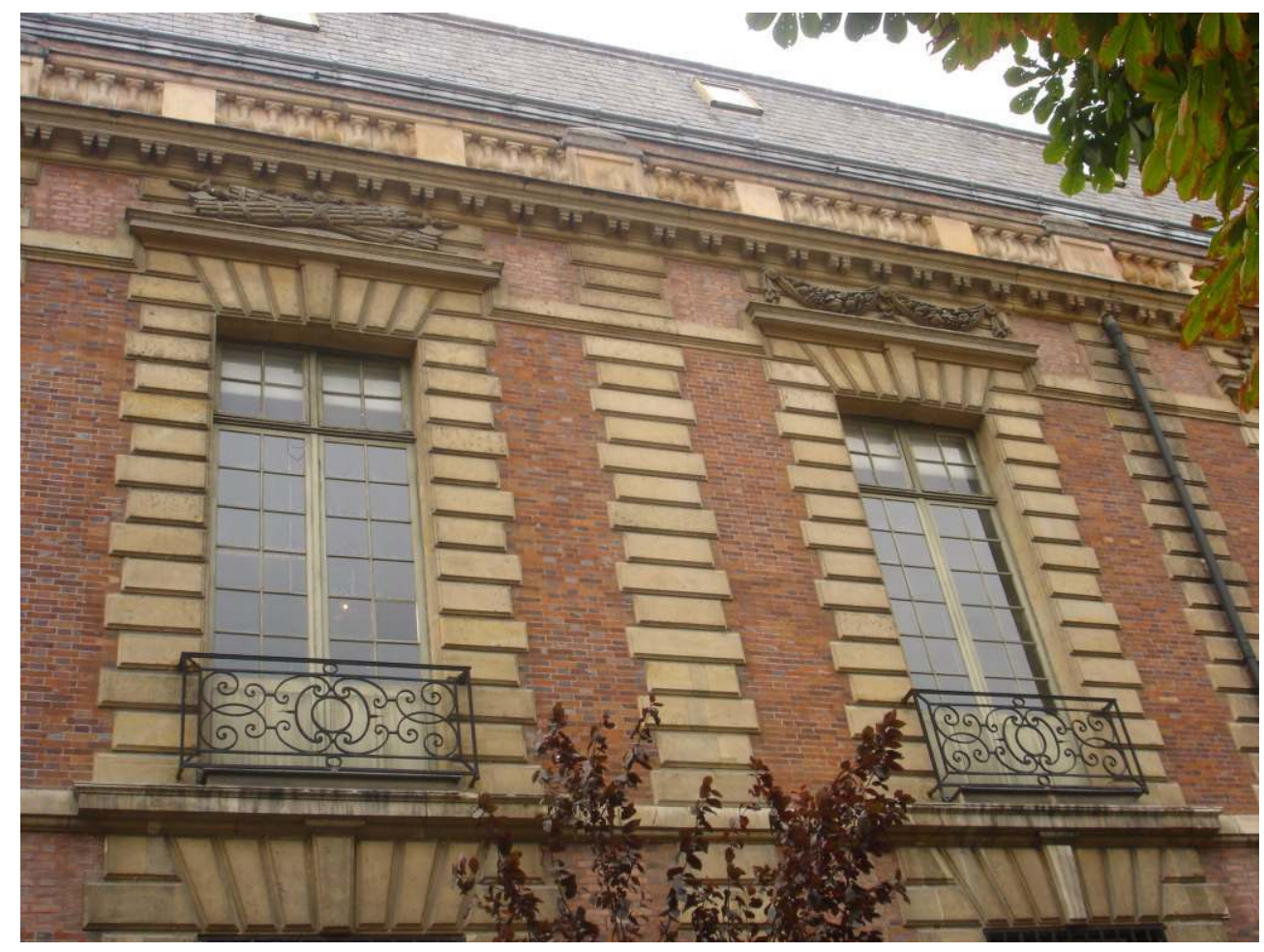

François Mansart. 1645

$\mathrm{Cl}$. auteur.

26 Les travaux relatifs à la reconstruction du comble de la galerie Mazarine commencés en 1868 furent poursuivis pendant l'hiver 1869. Sur le jardin Vivienne, la couverture présenta un brisis en ardoises de grand format fixées au crochet sur des liteaux métalliques et un terrasson en zinc, ensemble supporté par la charpente métallique ; sur la partie arrière, entre le terrasson et la salle Labrouste, le zinc fut choisi pour éviter les bris de verre dus aux éventuelles chutes d'ardoise (ill. 8).

\section{8 : Couverture de la galerie Mazarine côté jardin et côté salle de travail}

Éd. 1850-1940. Dessin aquarellé sur papier. 622 × 785 mm Arch. adm. B.n.F., 2011/001/4055.

27 La console terminant le chéneau de la galerie Mazarine fut posée le 17 décembre 1869.

28 La restauration des peintures de la galerie Mazarine ne faisait pas partie du projet initial de Labrouste :

«Le moment me paraît venu de songer aux raccords à faire aux peintures qui décorent l'intérieur de cette galerie. Je n'ai jamais songé à rétablir les peintures dans tout leur état primitif ; il ne s'agit selon moi que de raccorder les ornements entre eux et réparer les déchirures qui existent sur les angles de la galerie. Il en serait de même pour les peintures qui décorent les parties verticales des murs : les peintures qui ne sont point de la main de Romanelli comme la voûte, sont fort détériorées et quelques-unes ont complètement disparu ${ }^{58}$. »

Pour restaurer les paysages de Grimaldi qui décorent les niches, Labrouste propose le peintre Alexandre Desgoffe (1805-1882) qui avait décoré en 1864 la grande salle de lecture de la bibliothèque de motifs d'arbres et de ciel. Le prix de $4500 \mathrm{fr}$. qui lui est proposé 
alors ne concerne que la réparation des paysages et ne s'applique pas à la restauration des figures à fresque de Romanelli qui ne doivent être l'objet d'aucunes retouches ni réparations ${ }^{59}$ (ill.9 et 10$)$.

\section{9 : Jupiter foudroie les géants}

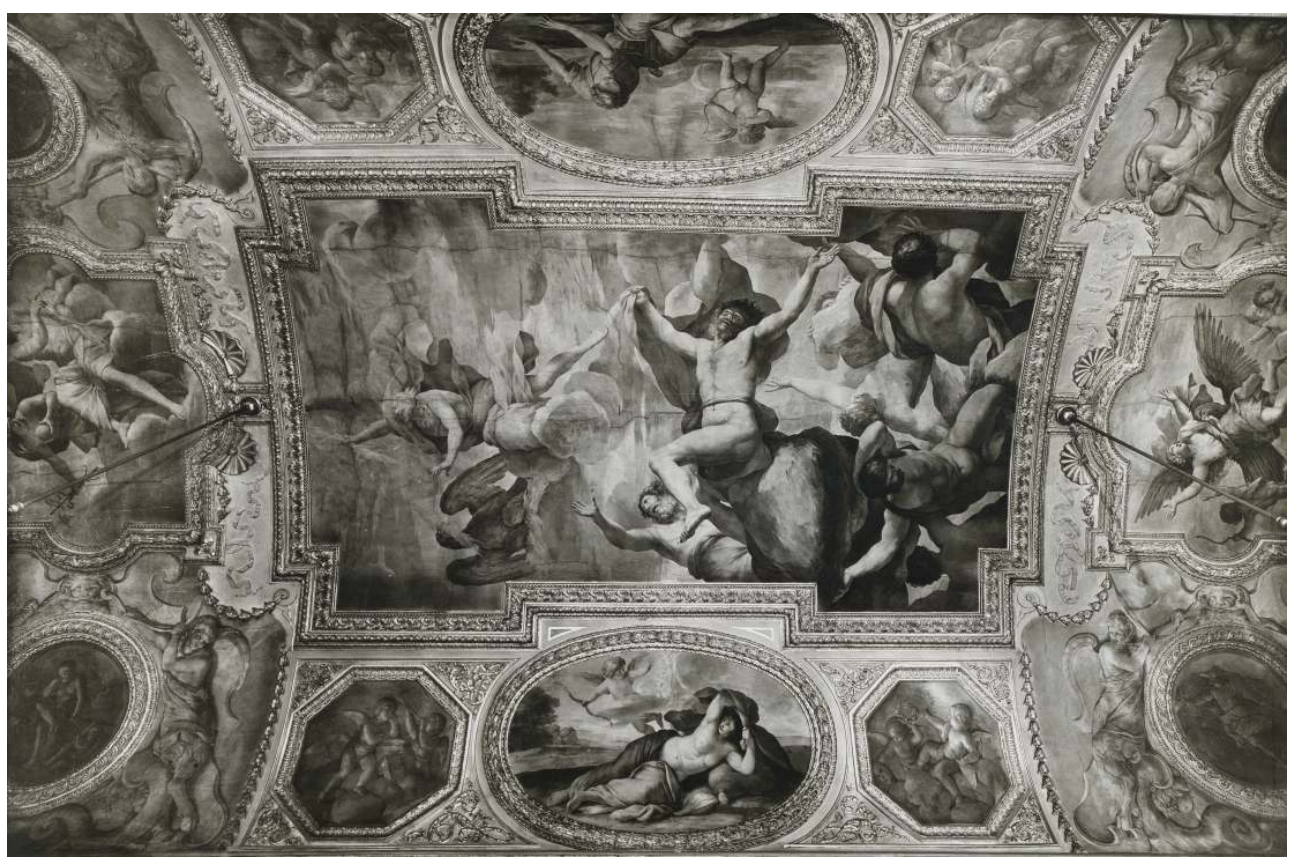

Giovanni Francesco Romanelli. Peinture centrale du plafond de la galerie Mazarine avant la restauration de 1975 (telle qu'elle avait été remaniée au XIX siècle)

B.n.F., département des estampes, VA237 (9) Fol H23658. 


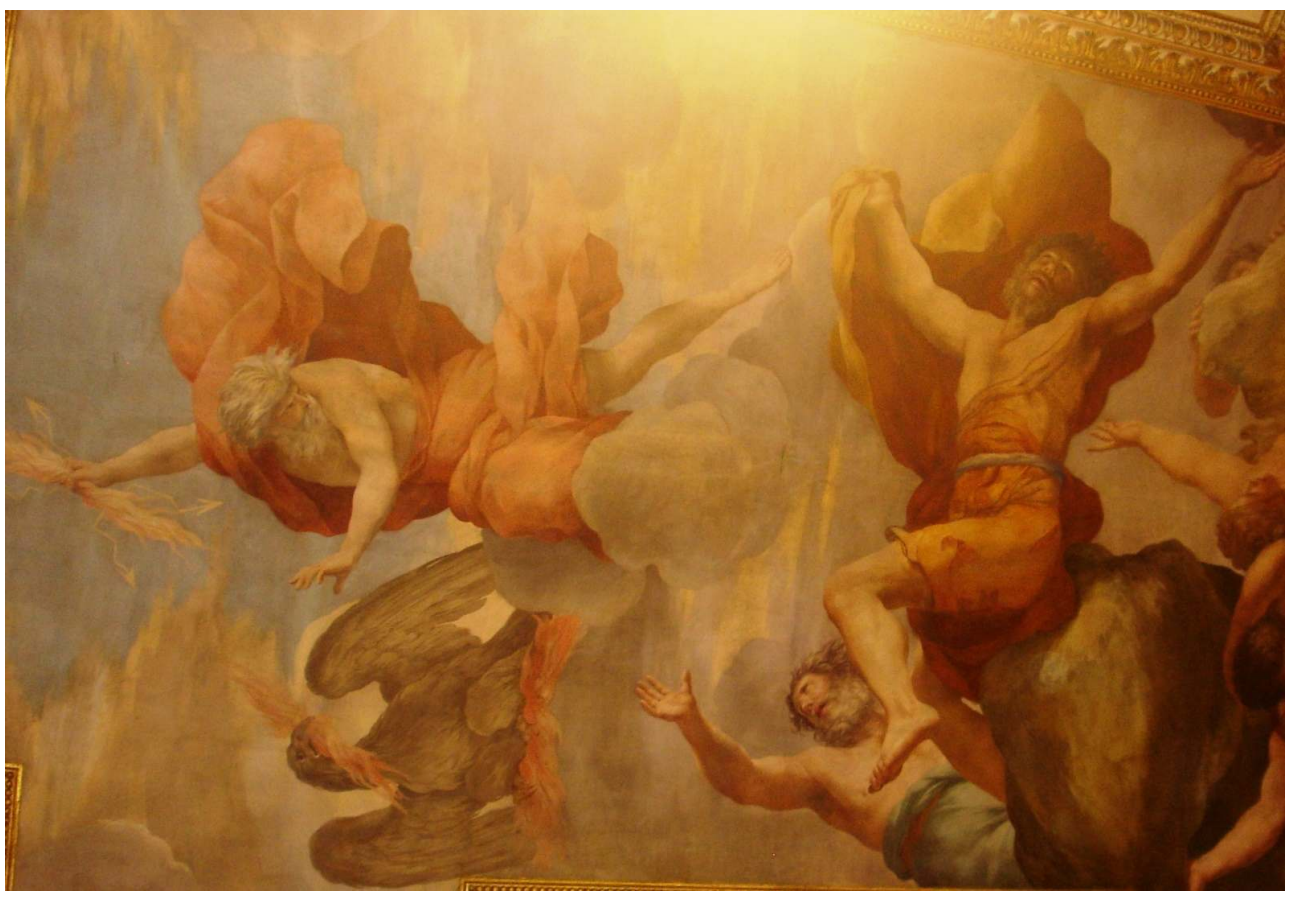

Giovanni Francesco Romanelli. Peinture centrale du plafond de la galerie Mazarine, état initial et actuel. Photo du 5 septembre 2012

Cl. auteur

Cette restauration est qualifiée par Labrouste de $"$ remarquable $»^{60}$. Pour restaurer les peintures décoratives des niches et ébrasements de fenêtres de la galerie Mazarine, on choisit le peintre décorateur Alexandre Denuelle ${ }^{61}$. Le nettoyage de la façade de la galerie Mazarine sur la rue Vivienne commence le 28 octobre 1870. Le comble de la galerie entièrement reconstruit en fer est garni de casiers et de tablettes destinés à recevoir une grande partie des livres du département des manuscrits avec lequel ce comble communique directement par un escalier intérieur également en fer. La porte qui permet d'accéder à l'escalier est percée le 14 décembre 1869. Dans la galerie Mazarine, Félix Perin, sous-inspecteur à la bibliothèque, fait enlever les tablettes qui servaient à ranger les manuscrits ${ }^{62}$; elles sont numérotées et rangées. Les parois verticales de la salle sont recouvertes d'une toile marouflée peinte d'après la décoration murale représentée sur le portrait gravé en 1659 par Robert Nanteuil et Pierre Van Schuppen représentant le cardinal Mazarin dans la galerie haute.

31 La phase suivante sera la démolition de l'hôtel de Chevry. Les nouveaux bâtiments qui longent la rue des Petits-Champs s'achèvent sur une rotonde d'angle: le «Pavillon Voltaire $"^{63}$. Les travaux sur la rue de Richelieu se poursuivent pendant l'année 1860 jusqu'à la porte principale (qui n'est pas à sa place actuelle) ; l'espace est délimité pour la salle de travail et le magasin central des imprimés ${ }^{64}$. Le département des imprimés entre en possession des bâtiments sur la rue Neuve-des-Petits-Champs. L'année 1867 marque la fin des travaux ; la grande salle de lecture pour quatre cents personnes ${ }^{65}$ est ouverte le 12 juin 1868, le grand comble de la rue de Richelieu achevé en 1873, le magasin de réserve en $1875^{66}$. 

par une double arcature formant galerie couverte, permettant de voir du dehors, la fontaine et les plates-bandes prévues dans la cour d'honneur, l'arcade du milieu étant l'entrée principale de la Bibliothèque. Dans les arcades latérales devaient se dresser les statues des plus illustres bibliophiles. Des préoccupations sécuritaires firent abandonner ce projet $^{67}$. De 1868 à 1875 , Henri Labrouste rase ce qui restait de la grande galerie de l'hôtel de Nevers au nord de la porte d'entrée, et élève à la place une façade néo-classique sur la place Louvois. Elle est alourdie par le profil des combles où sont logés les réservoirs d'eau ${ }^{68}$. Charles-Marie Widor, secrétaire perpétuel de l'Académie des beaux-arts, ami de Jean-Louis Pascal, ne l'aimait guère : « Nous n'avons plus, sous les yeux, de ce côté-là, que l'amorphe, indigeste gâteau d'un édifice massif, lequel n'est à vrai dire, qu'un mur qui voudrait être grec et s'afflige de rester laid $»^{69}$.

1873, l'entrée principale de la Bibliothèque est déplacée vers le nord de la rue de Richelieu ${ }^{70}$; en 1874, Henri Labrouste commence à démolir le bâtiment qui ferme au nord la cour d'honneur (ill. 11).

\section{11 : Plan du rez-de-chaussée de la Bibliothèque nationale à la mort d'Henri Labrouste (1875)}

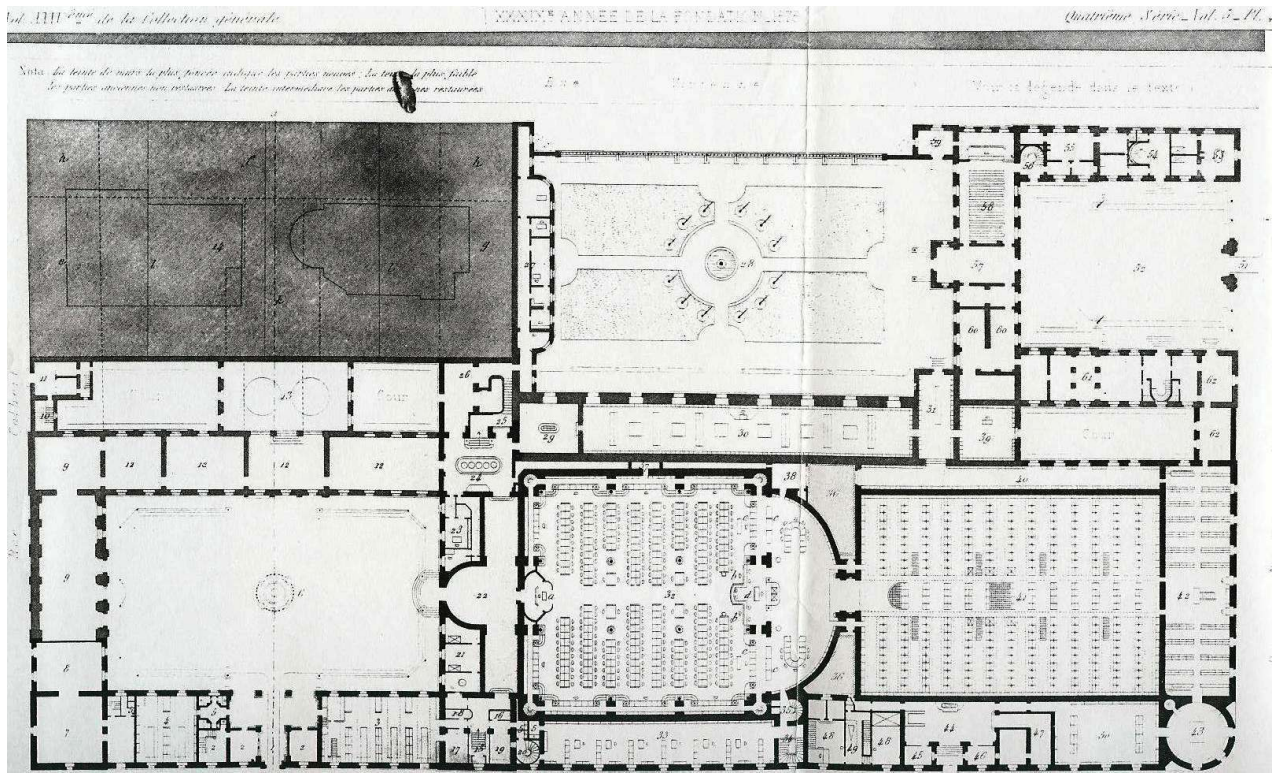

Léon Labrouste, « Bibliothèque nationale, à Paris, par M. H. Labrouste, membre de I'Institut », R.G.A.T.P. 1878, pl. 40.

\section{Jean-Louis Pascal, architecte de la Bibliothèque, premiers travaux (1875-1878)}

Jean-Louis Pascal poursuit donc tout d'abord l'œuvre de son illustre prédécesseur :

« L'œuvre de reconstruction de la Bibliothèque nationale a comporté bon nombre d'innovations, de dispositions ingénieuses, qui n'ont pas peu contribué à la grande réputation de mon prédécesseur, $M$. Labrouste. Je n'aurai pendant plusieurs années encore qu'à m'inspirer de sa pensée primitive, sans négliger peut-être les améliorations de détail que l'usage aurait pu me signaler, mais en n'abandonnant 
pas le plan d'ensemble adopté à l'origine et poursuivi avec une rare persistance. Disposition de casiers, agencements, planchers à claire-voie, mobiliers, bureaux, tables, pupitres, vitrines, tout ce que j'ajoute ou que je transforme dans les salles qui me sont confiées, je le maintiens conforme aux indications que me donnent les dessins restés à l'agence, les modèles existants, et souvent les documents fournis par les entrepreneurs même, car le bureau de l'architecte a été brûlé une fois, il y a longtemps, et de nombreux dessins ont été perdus dans l'incendie, qui sont en double chez eux $»^{72}$. juillet-août 1875, une exposition de monuments épigraphiques. En 1876, Jean-Louis Pascal demande l'attribution de crédits pour installer un mobilier définitif dans la galerie Mazarine ${ }^{81}$.

\section{La galerie Mazarine est transformée en salle d'exposition}

Pour préparer l'Exposition universelle de 1878, l'administrateur général de la Bibliothèque Léopold Delisle demande à Jean-Louis Pascal d'approprier la galerie Mazarine en une sorte de musée ${ }^{82}$. La reconstitution de la salle dans son état ancien est alors jugée «nécessaire du point de vue de la conservation d'un monument historique et artistique ${ }^{83}$. Jean-Louis Pascal propose un aménagement correspondant à celui fait au Louvre dans la galerie d'Apollon ou le musée Campana: «J'aurais voulu faire ce travail pour l'exposition universelle ; l'argent me manquera, et nous ne ferons peut-être que des meubles en sapin ; toujours du provisoire, comme vous voyez, mais ce qui est nécessaire, cependant, pour ne pas trop négliger de faire notre partie dans la solennité internationale ${ }^{84}$.

Sur les parois de la salle, entre les fenêtres et les niches, il propose de placer dix-huit vitrines assez basses (ill. 12) encadrées par des gaines supportant des bustes et de placer au milieu de la salle, dans la longueur, en laissant de chaque côté un passage de deux mètres, quatre tables basses ${ }^{85}$ surmontées de pupitres garnis de glaces, tables séparées de distance en distance par des statues ou objets curieux ${ }^{86}$. 


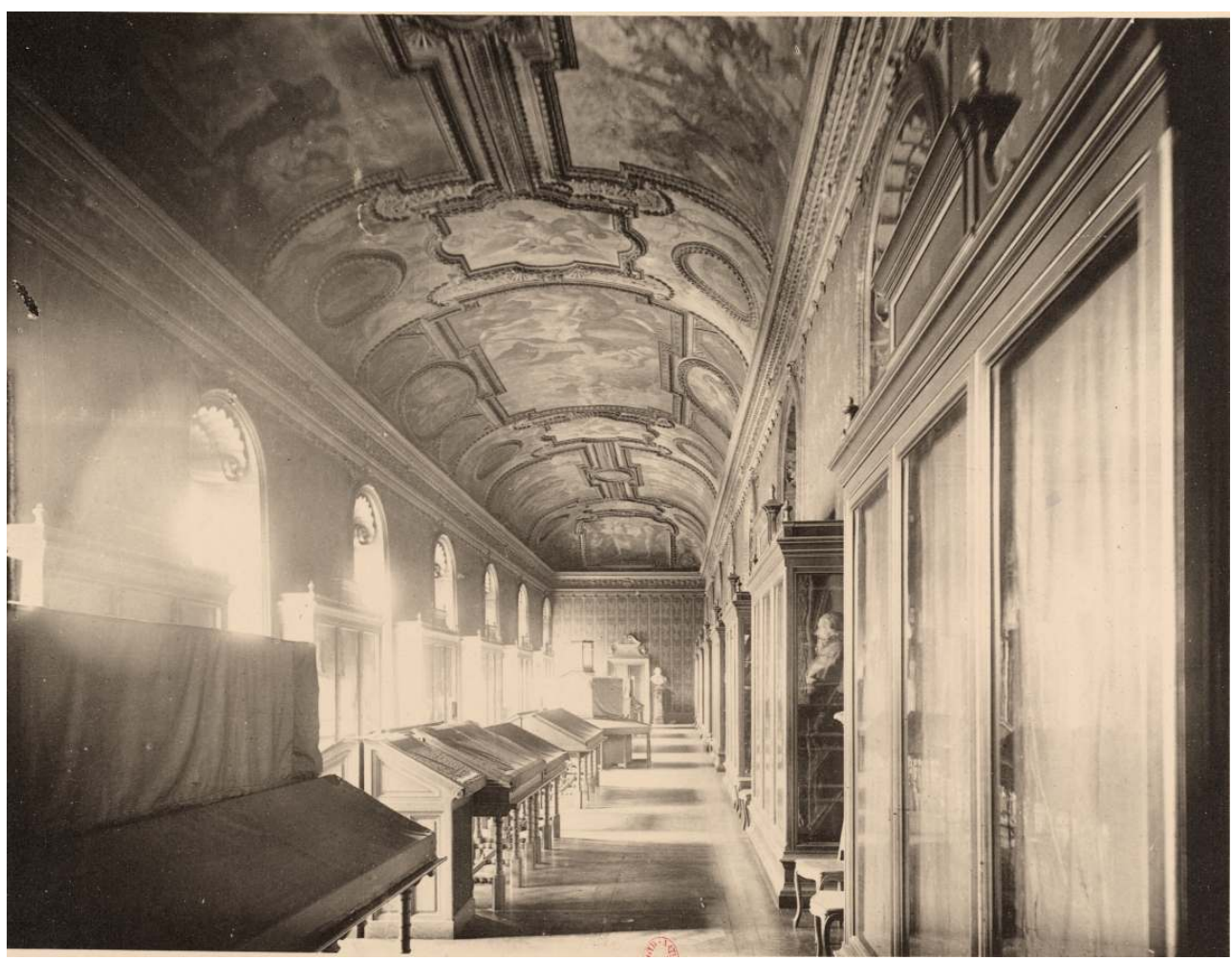

1645. Peintures de Romanelli et Grimaldi. Au $7^{\text {er }}$ étage, l'ancienne galerie des peintures du Cardinal, transformée par Jean-Louis Pascal en salle d'expositions puis en salle de travail du département des estampes

Arch. adm. B.n.F., 2007/061/001, pl. 109

41 À cet effet, la commission du vase de Sèvres met au concours en 1879 un objet décoratif destiné à la galerie Mazarine (la hauteur du vase ne doit pas dépasser $1,80 \mathrm{~m}$.) et l'administration des beaux-arts demande de disposer des parois de l'antichambre carrée qui précède cette galerie (salle du Parnasse improprement appelée parfois «chambre de Mazarin ») pour des tapisseries des Gobelins ${ }^{87}$, à mettre aussi au concours ${ }^{88}$. Les cartons de ces trois tapisseries ${ }^{89}$ en haute lisse, sont réalisés par le peintre François Émile Ehrmann $^{90}$ (1833-1910), lauréat du concours de $1879^{91}$. Elles symbolisent trois grandes époques de la civilisation : l'Antiquité, le Moyen Âge et la Renaissance. Il s'agit de : Les Lettres, les Arts et les Sciences dans l'Antiquité ${ }^{92}$ (ill. 13), Le Manuscrit ${ }^{93}$ et L'Imprimég ${ }^{94}$, ces deux derniers panneaux se trouvant de part et d'autre de la fenêtre située sur le mur est de l'antichambre ${ }^{95}$. 


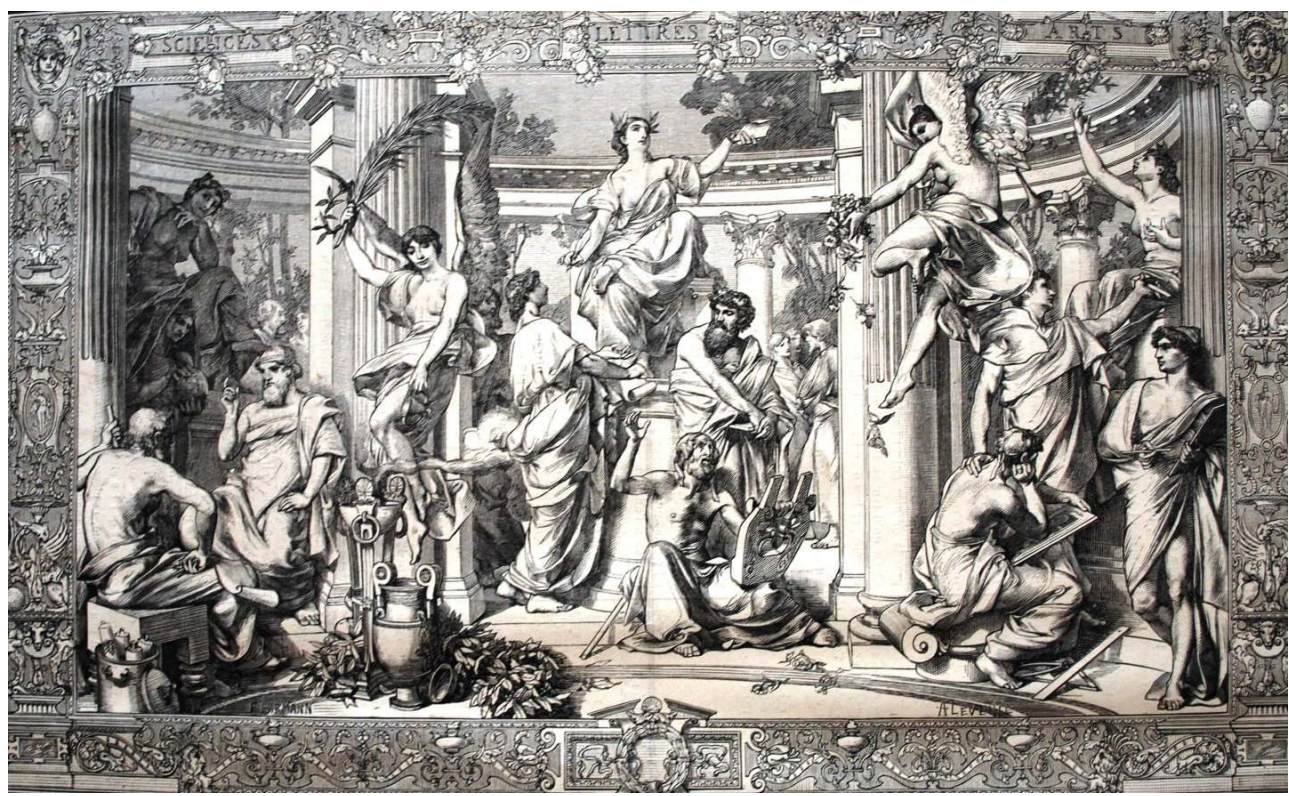

Tapisserie présentée au salon de 1880. Carton de François Ehrmann. Mur ouest de la salle du Parnasse (antichambre au nord de la Galerie Mazarine)

B.n.F., département des estampes, VA 237 Fol. H23632.

42 Au milieu de la pièce s'élève alors un monument sculpté en 1718 par le sculpteur Louis Garnier (1639-1728), élève de François Girardon (1628-1715), modèle d'un projet commandé par l'homme de lettres Évrard Titon du Tillet (1677-1762), représentant le «Parnasse français », sorte de trophée de bronze en forme de pyramide ${ }^{96}$, à la gloire de Louis XIV et des plus célèbres poètes et musiciens depuis François $1^{\text {er }}$, que Pascal fit remonter et restaurer par l'entreprise Barbedienne ${ }^{97}$ quelques années après son arrivée à la Bibliothèque ${ }^{98}$; Louis XIV en Apollon s'y tient près du sommet, que couronne Pégase avec la Nymphe de la Seine, les trois Grâces étant incarnées par $\mathrm{M}^{\text {mes }}$ de la Suze, Deshoulières et $\mathrm{M}^{\text {elle }}$ de Scudéry ; plus bas, sont représentés Corneille, Racine, Molière, Segrais, La Fontaine, Chapelle, Boileau, Crébillon, Voltaire, Jean-Baptiste Rousseau et Lulli ; vingt-deux génies ailés forment divers groupes. En bas du monument, Titon du Tillet, debout, tient la dédicace de son œuvre ${ }^{99}$.

43 La galerie sera ornée à partir de $1882^{100}$ de bustes des personnages dont le nom est intimement lié à l'histoire de la Bibliothèque nationale ${ }^{101}$ : Charles $\mathrm{V}$, François $1^{\mathrm{er}}$, Nicolas Claude Fabri de Peiresc ${ }^{102}$ (1580-1637), Michel de Marolles ${ }^{103}$ (1600-1681), Guillaume Budé, Van Praët ${ }^{104}$ (1754-1837), Louis XIV, Colbert, Pierre Dupuy ${ }^{105}$ (1582-1651), Jaques Auguste de Thou $^{106}$ (1553-1617), le comte de Caylus ${ }^{107}$ (1692-1765), Dom Mabillon (1632-1707). Ils remplacent des modèles en bois posés provisoirement lors de l'exposition de 1878. Pascal voulait des sculpteurs de renom pour effectuer ce travail: Delaplanche, Ernest Eugène Hiolle $^{108}$ (1833-1886), Charles Degeorge, Louis Maximilien Bourgeois ${ }^{109}$ (1839-1901), Barrias, Mercié, Louis Noël ${ }^{110}$ (1839-?), Allar, Jules Isidore Lafrance ${ }^{111}$ (1841-1881), Laurent Honoré Marqueste ${ }^{112}$ (1848-1920) : «Le choix des personnages représentés aussi bien que le caractère décoratif de la salle justifiant l'emploi de costumes mouvementés et d'une exécution coûteuse, il me paraît que le prix à affecter à chacune de ces figures devrait être de 3400 francs si l'État fournissait le marbre et de 4000 francs dans le cas contraire " ${ }^{113}$. Le sous-secrétaire d'État aux beaux-arts choisit Emmanuel Frémiet ${ }^{114}$ (1824-1910) pour 
sculpter le buste de Charles V, Pierre Jules Cavelier pour celui de François $1^{\mathrm{er}}$, Yselin pour celui du comte de Caylus, Jean Antonin Injalbert ${ }^{115}$ (1845-1933) pour celui de Van Praët, et Laurent Honoré Marqueste pour celui de Jacques Auguste de Thou ${ }^{116}$. Les modèles des bustes de Peiresc, Mabillon, l'abbé de Marolles et Colbert, sont commandés à Charles Vital Cornu (1851-1927), Albert Guilloux ${ }^{117}$, Émile Hugoulin ${ }^{118}$ (1848-1923) et Honoré Icard ${ }^{119}$. Le modèle du buste de Guillaume Budé est commandé à Maximilien Bourgeois ${ }^{120}$ et celui de Pierre Dupuy à Vital-Dubray ${ }^{121}$.

Des gaines en marbre poli de brèche violette, de vert de mer, de campan rouge, seront commandées par la suite à l'entrepreneur Drouet Langlois pour supporter les bustes ${ }^{122}$. Le dernier buste réalisé, celui de Louis XIV par Jean Jules Cambos ${ }^{123}$ (1828-1917) est déposé au dépôt des ouvrages d'art du palais des Champs-Élysées le 7 mai 1888.

\section{Création d'un atelier de photographie}

Un atelier photographique (ill. 14) était devenu indispensable par suite des donations faites par des établissements ou des gouvernements étrangers qui réclamaient une réciprocité pour la reproduction de documents uniques à la Bibliothèque qui manquaient à leurs collections ${ }^{124}$.

\section{14 : Atelier de la photographie. Bibliothèque nationale. Projet de Jean-Louis Pascal}

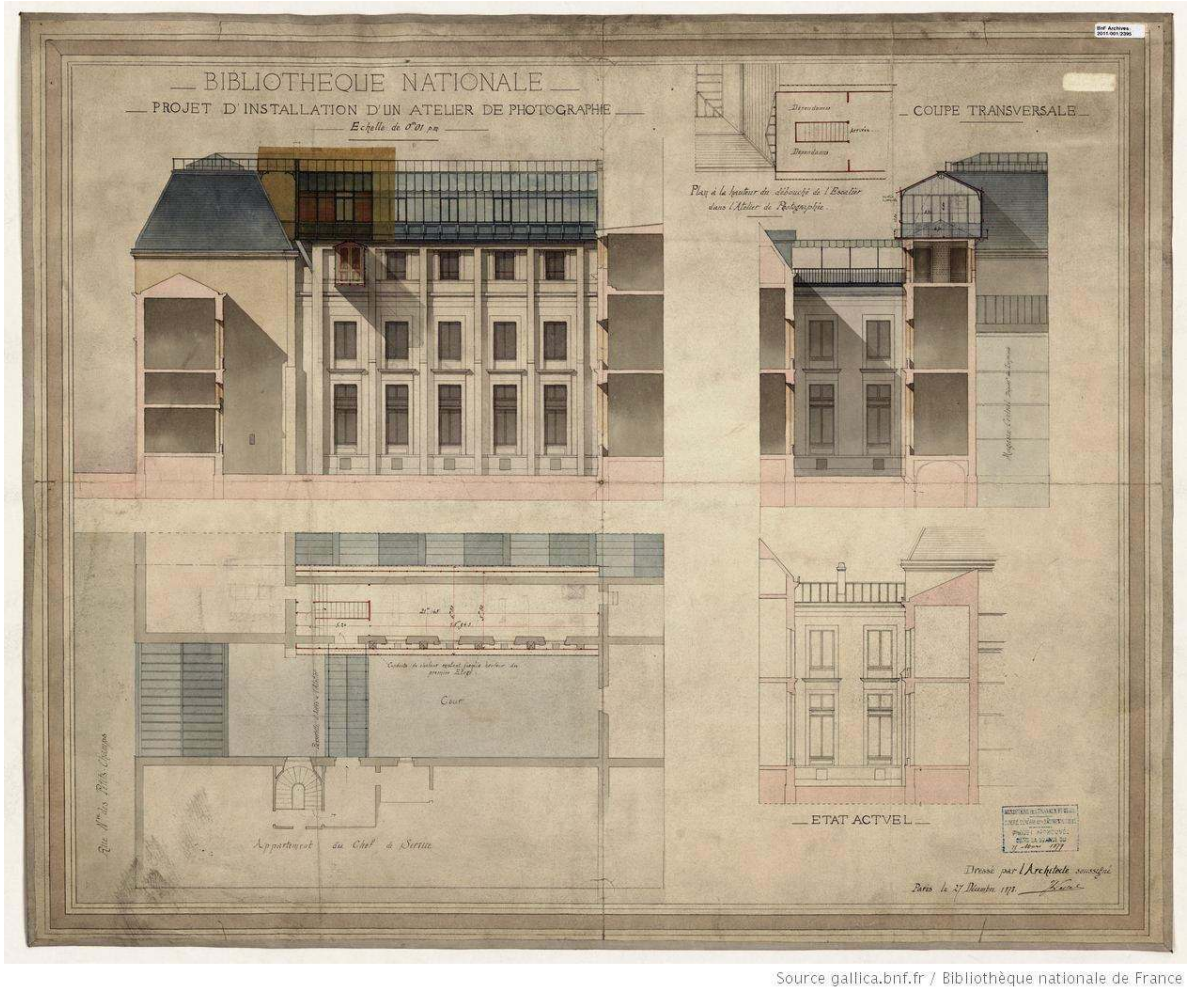

Plan, coupe et élévation. Dessin aquarellé sur papier. 650 × 797 mm. 27 décembre 1878 Arch. adm. B.n.F., 2011/001/797

Pascal propose de construire cet atelier au-dessus d'un corps de bâtiment étroit bordant le magasin central à l'est ${ }^{125}$ et renfermant au rez-de-chaussée une dépendance des estampes (ill. 15) et au $1^{\mathrm{er}}$ étage la galerie de la géographie. 


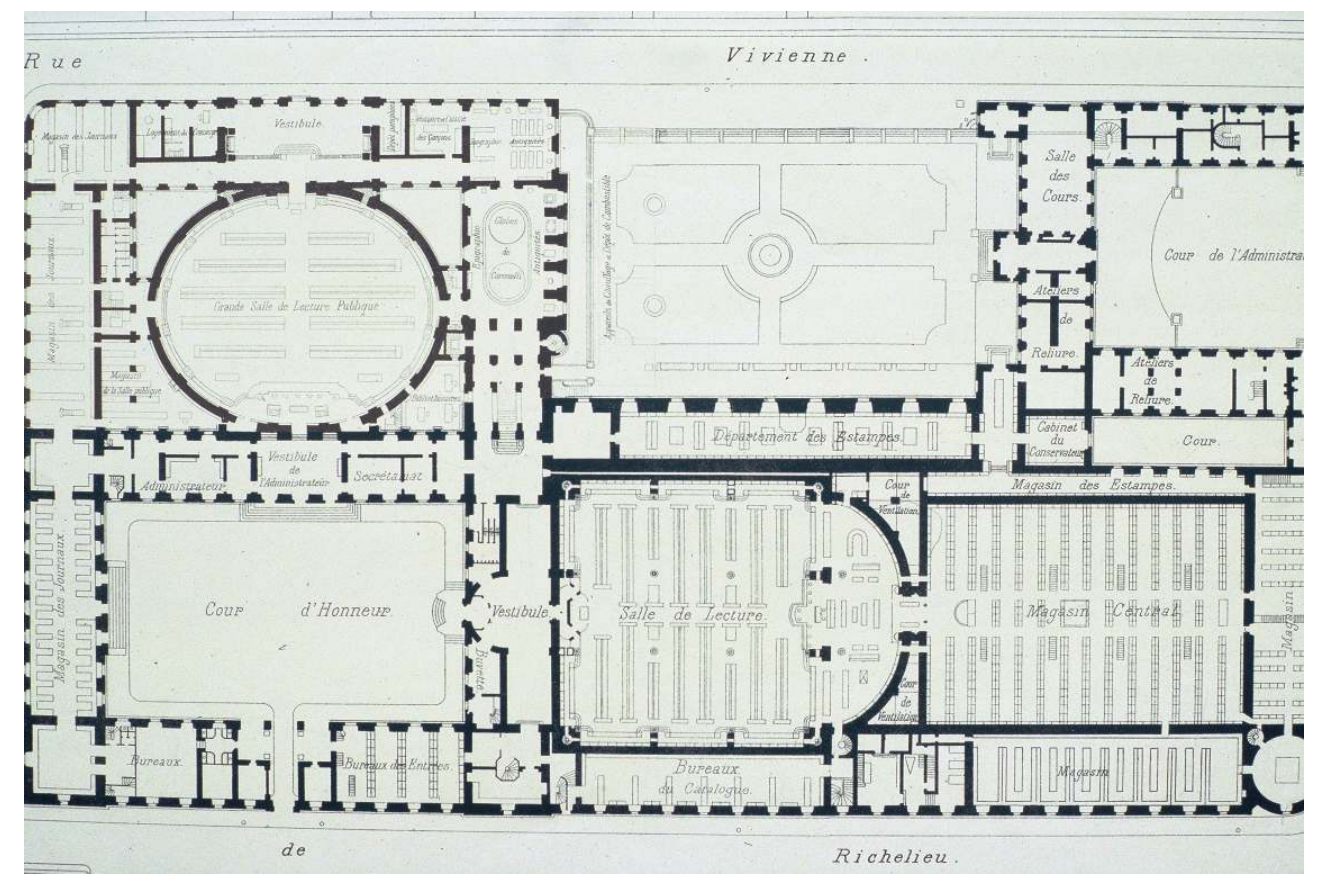

19 janvier 1892

Reproduit dans L'Architecture, 1933, vol. XLVI, n 1, p. 10

Le bâtiment est couvert par un toit en appentis à la hauteur du comble de la galerie Mazarine et de celui du département des manuscrits ${ }^{126}$. Une communication entre ces deux parties de l'édifice serait très précieuse pour la facilité des services: «Je propose donc [dit Pascal] de profiter de la nécessité d'élever autant que possible l'atelier photographique afin de le mettre à l'abri des reflets et des ombres portées, pour établir un nouvel étage de communication, interposé entre la galerie de la géographie et l'atelier $127 »$.

Pascal propose ou bien d'établir un escalier spécial dans la cour de la reliure ou bien de se servir de l'escalier existant (dans l'hôtel Tubeuf donnant sur la rue des Petits-Champs) et de franchir la cour par une passerelle à la hauteur du comble. C'est cette dernière solution qui est préférée, pour respecter la surface très restreinte de la petite cour sur laquelle donnent au rez-de-chaussée le bureau de Delaborde et les magasins des estampes (ill. 15) et au $1^{\text {er }}$ étage, les dépôts des manuscrits, la salle de géographie, les appartements : «Cette passerelle serait dallée en verre et couverte de même pour enlever le moins de jour possible [...] Une charpente provisoire devra être élevée au-dessus de la galerie à construire afin de ne pas interrompre le service de la géographie et de parer les éventualités d'inondation dans la salle du $1^{\mathrm{er}}$ étage $»^{128}$.

En décembre 1876, faisant le point sur les travaux accomplis, Jean-Louis Pascal écrit :

«L'année prochaine, j'aurai livré à la Bibliothèque un corps de bâtiment complet sur la place Louvois, avec son ameublement; deux calorifères y fonctionneront continuellement l'hiver. Le magasin central, qui, jusqu'ici, n'avait pas été chauffé, vient d'être pourvu d'un appareil puissant [...] Le cabinet des médailles prendra possession dans quelques mois de locaux non chauffés jusqu'ici, d'une part sur la rotonde, d'autre part sur la réserve actuelle des imprimés ${ }^{129}$. 
effet, à partir de 1878, Pascal déménage le département des médailles vers les bâtiments construits par Labrouste entre la rotonde Voltaire et l'hémicycle de la salle de travail, le long de la rue de Richelieu. Labrouste avait détruit en 1865 l'ancien cabinet des médailles placé dans l'arcade Colbert, à l'angle nord-ouest de la Bibliothèque. Le legs par le duc de Luynes, trois ans auparavant, de son abondante collection, legs assorti d'une clause exigeant d'en conserver l'unicité, avait entraîné cette destruction.

Léopold Delisle propose alors à Pascal de placer la statue de Voltaire dans le pavillon précédant la future galerie de la réserve :

«Elle regarderait cette galerie et serait adossée au futur magasin du département des médailles. Dans cette hypothèse, les jours d'ouverture au public, les visiteurs pourraient se promener, au $1^{\text {er }}$ étage, dans la galerie Mazarine, sur le pont de bois ${ }^{130}$ et dans le pavillon faisant suite au pont de bois. De ce pavillon, ils auraient la perspective de la galerie de la réserve. Nous affecterions les armoires du pavillon à la collection voltairienne de Beuchot, à la collection Payen, relative à Montaigne, et aux collections Fontanieu et Morel de Thoiry. $»^{131}$

En 1877, Pascal insère dans son budget ${ }^{132}$ le commencement des travaux de reconstruction des anciens bâtiments sur la rue Colbert. Ces premiers grands travaux de Pascal à la Bibliothèque sont dans la suite de ceux de Labrouste, autour de la cour d'honneur. Labrouste avait envisagé une reconstruction totale des bâtiments et l'avait même amorcée au nord de la cour d'honneur ${ }^{133}$. « Au contraire de Labrouste, Pascal était le respect même ${ }^{134}$. Léon Labrouste, fils du précédent architecte n'écrivait-il pas : "L'œuvre commencée par M. Labrouste ne pouvait être confiée à des mains plus habiles, à un artiste plus respectueux de la mémoire de son illustre prédécesseur ${ }^{135}$.

\section{NOTES}

1. Pascal est aussi en charge de l'entretien du Monument équestre de Louis XIV par François Joseph Bosio (1768-1845) qui orne la place des Victoires à Paris $\left(2^{\mathrm{e}}\right)$ depuis 1822.

2. Léopold Delisle est administrateur général de la Bibliothèque nationale en 1874, Henry Marcel en 1905, Théophile Homolle en 1913, Pierre Roland-Marcel en 1923.

3. Gustave Adolphe Gerhardt (1843-1921), alors inspecteur des travaux de réfection de l'ancienne bibliothèque du Louvre, incendiée pendant la Commune, révèle à Pascal que Lefuel lui a proposé de faire, sur le lot de Pascal, un travail » tout mâché et préparé par lui [Pascal] depuis longtemps ", sous prétexte que Gerhardt a les fonds disponibles et que Pascal n'en a plus sur son crédit. «Ce dernier point étant inexact, je flaire quelque moyen de me mettre à l'arrière-plan, que je redoute fort. [...] Depuis trois ans je fais de la maçonnerie, de la surveillance de chantier, d'attachements et de comptabilité ; je n'ai nulle occasion de montrer ce par quoi je vaux peut-être quelque chose. Peut-être pour mon chef semblé-je ne rien produire, parce que mon temps se passe sur le tas à des besognes fastidieuses. Je crains d'autant plus de lui qu'il ne m'a jamais soufflé mot de griefs qu'il a peut-être contre moi et que je n'ai jamais appris quelque mécontentement 
de sa part que par des voies absolument détournées ». B.E.B.A., Ms 744, Lettres de l'architecte Jean-Louis Pascal à Charles Garnier, op. cit., lettre nº 73, 24 juin 1875.

4. Le comte Étienne de Cardaillac, directeur des bâtiments civils.

5. B.E.B.A., Ms 744, Lettres de l'architecte Jean-Louis Pascal à Charles Garnier, op. cit., lettre n -74, 29 juin 1875.

6. Henri-Paul Nénot, «La vie et les œuvres de Jean-Louis Pascal », L'Architecture, 25 septembre 1921, p. 13-14.

7. Voir l'histoire si complète qu'en a retracée le comte Léon de Laborde dans: De l'organisation des bibliothèques dans Paris, $4^{e}$ lettre: Le palais Mazarin et les habitations de ville et de campagne au XVII siècle, Paris, A. Franck, 1845, 408 p.

8. Léon Labrouste, La Bibliothèque nationale, son début et ses accroissements, ses bâtiments et ses constructions, ses agrandissements, ses travaux, Paris, H. Luthier, 1885, 94 p., p. 11.

9. Ursula Baurmeister et Marie-Pierre Laffitte, « De l'unité au partage », Bruno Blasselle et Laurent Portes (dir.), Mélanges autour de l'histoire des livres imprimés et périodiques, Paris, B.n.F., 1998, 369 p., p. 37-63.

10. Emmanuel Le Roy Ladurie, «Au commencement était la Librairie du Roi », Bibliothèque de France, premiers volumes, I.F.A., éd. Carte Segrete, Paris, 1989, 274 p., p. 25-28.

11. C'est l'actuel lycée Louis-le-Grand, 123 rue Saint-Jacques dans le Ve arrondissement de Paris.

12. Bernard Marrey, "L'homme, le livre, la lumière ", Bibliothèque de France, premiers volumes, op. cit., p. 29-59. C'est l'actuelle École pratique de la faculté de médecine de Paris, 15 rue de l'École de Médecine (6 $\left.6^{\mathrm{e}}\right)$. Seul subsiste le réfectoire de l'ancien couvent.

13. Arch. nat., $\mathrm{F}^{21} 5854$, ministère des travaux publics, achèvement de la Bibliothèque nationale, rapport de Paul Deschanel, député, Paris, 1892.

14. Henri Bouchot, «Les derniers travaux de décoration exécutés à la Bibliothèque nationale par M. Pascal, architecte ", Revue des arts décoratifs, $12^{\mathrm{e}}$ année, 1891-1892, p. 81-97. Henri Bouchot était conservateur du département des estampes à la B.N.

15. Il était situé au rez-de-chaussée de la partie ouest de l'aile sud donnant sur la cour carrée.

16. François Fossier, Les dessins du fonds Robert de Cotte de la Bibliothèque nationale de France, architecture et décor, Paris, Rome, B.n.F., École française de Rome, 1997, 795 p., p. 129, 133.

17. Jean-François Foucaud, » Extensions et travaux de la Bibliothèque nationale ", Histoire des bibliothèques françaises. Les bibliothèques de la Révolution et du XIXe siècle; 1789-1914, sous la direction de Dominique Varry, Paris, 1991, éd. du Cercle de la Librairie, 671 p., p. 335-354.

18. Louis Battifol, « Les origines du Palais Mazarin », Gazette des beaux-arts, t. XXXIX, 1915, p. 265-289.

19. Claude Dulong, « Du nouveau sur le palais Mazarin : l'achat de l'hôtel Tubeuf par le Cardinal », Bibliothèque de l'École des chartes, tome 153, Première livraison, janvier-juin 1975, Paris-Genève, Librairie Droz, 1995, 250 p., p. 131-155.

20. Aux deux bouts de la terrasse surmontant la porte cochère et les écuries, Mansart avait édifié deux lanternons couverts de petits dômes, pour loger les escaliers.

21. La galerie Mazarine abrite les collections de sculpture du cardinal Mazarin au rez-dechaussée et ses collections de peinture à l'étage. 
22. D'où son nom d'Hôtel de Nevers. L'autre partie, l'ancien hôtel Tubeuf, revint à la sœur de Philippe, Hortense, qui avait épousé le marquis de Meilleraye, duc de Mazarin après la mort du Cardinal. Il fut vendu en 1719 à la Compagnie des Indes.

23. Le rez-de-chaussée voûté contenait des écuries de $54 \mathrm{~m}$. de long sur $8 \mathrm{~m}$. de large. Louis Battifol, «Les origines du Palais Mazarin », Gazette des beaux-arts, op. cit., p. 281.

24. Elle faisait $60 \mathrm{~m}$. de long, $9 \mathrm{~m}$. de large, $8 \mathrm{~m}$. de haut.

25. Louis Battifol, « Les origines du Palais Mazarin », op. cit., p. 282.

26. Il semble que la marquise de Lambert l'aurait revendu au roi pour une somme de 500000 livres, le 13 juin 1724, à condition d'en garder la jouissance durant sa vie et celle de son fils. Léon Labrouste, La Bibliothèque nationale, son début et ses accroissements, ses bâtiments et ses constructions, ses agrandissements, ses travaux, op. cit., p. 26.

27. Cela correspond au $\mathrm{n}^{\circ} 27$ du plan de 1850 (ill. 3).

28. Cela correspond au $\mathrm{n}^{\circ} 26$ du plan de 1850 (ill. 3). La salle à manger était située au $\mathrm{n}^{\circ}$ 25 , le salon au $n^{\circ} 24$ et aux deux $1^{\text {res }}$ travées de la salle 6 , la chambre à coucher dans la $3^{\mathrm{e}}$ et $4^{\mathrm{e}}$ travée de la salle 6 , le cabinet de toilette dans la $5^{\mathrm{e}}$ travée de la salle 6 . La marquise avait en outre la jouissance d'une basse-cour dans la partie du jardin occupée aujourd'hui par les salles $n^{\text {os }} 8$ et 9 sur le plan et la cour attenante, et par conséquent un droit de passage dans la grande cour de l'hôtel de Nevers. Léon de Laborde, De l'organisation des bibliothèques dans Paris, $4^{e}$ lettre: Le palais Mazarin et les habitations de ville et de campagne au XVII siècle, op. cit., n. 628 p. 384.

29. Ibid., p. 102-103.

30. L'hôtel de Lambert abrita ensuite le cabinet des médailles. En 1865, Labrouste le détruira presque entièrement. L'amorce de l'arcade subsiste encore.

31. Léon de Laborde, De l'organisation des bibliothèques dans Paris, $4^{e}$ lettre: Le palais Mazarin et les habitations de ville et de campagne au XVII e siècle, op. cit., p. 105-106.

32. François Fossier, Les dessins du fonds Robert de Cotte de la Bibliothèque nationale de France, architecture et décor, op. cit., p. 135.

33. Françoise Bléchet, » D'un déménagement à l'autre », Bruno Blasselle et Laurent Portes (dir.), Mélanges autour de l'histoire des livres imprimés et périodiques, op. cit., p. 93-106.

34. Après le transfert de la bibliothèque, l'hôtel de Colbert et ses deux maisons de la rue Vivienne furent abattus et remplacés par les écuries du Régent.

35. Henri Bouchot, «Les derniers travaux de décoration exécutés à la Bibliothèque nationale par M. Pascal, architecte », op. cit., p. 90.

36. Ces détails sont importants car «ils s'appliquent aux travaux exécutés, conservés depuis, et qui forment encore aujourd'hui, la majeure partie de la nouvelle salle des manuscrits restaurée par Pascal », Ibid., p. 92. Le bâtiment du fond de la cour d'honneur fut restauré par Pascal en 1885 et transformé en salle de lecture du département des manuscrits.

37. Attribuée jusque-là au fils de Robert de Cotte, Jules-Robert (1683-1767), l'architecte véritable en serait Ange-Jacques Gabriel (1698-1782), d'après les dernières découvertes d'Alexandre Gady, professeur d'Histoire de l'art à l'université de Paris IV.

38. François Fossier, Les dessins du fonds Robert de Cotte de la Bibliothèque nationale de France, architecture et décor, op. cit., p. 136. 
39. Elle enjambe la rue Colbert percée en 1683. Le petit hôtel est au $n^{\circ} 12$ de la rue Colbert.

40. Pierre François Léonard Fontaine, Journal 1799-1853, t. 2 : 1824-1853, Paris, E.N.S.B.A., I.F.A., 1987, 1373 p., p. 722.

41. Jean-François Foucaud, « Extensions et travaux de la Bibliothèque nationale », Histoire des bibliothèques françaises, op. cit., p. 337.

42. La Révolution avait ouvert la bibliothèque plus largement mais le nombre croissant des lecteurs dû à la quasi-absence dans Paris de lieux d'accueil pour un grand public posait des problèmes : c'est ce manque qui va amener la création d'une salle publique de lecture ouverte à tous, en 1833. On y met à disposition vingt mille livres. Dès les premiers jours, on accueille jusqu'à cinq cents personnes par jour. Simone Balayé, » Les publics de la Bibliothèque nationale ", Histoire des bibliothèques françaises, op. cit., p. 329-333. Simone Balayé, Kinga Kantorska, » Une Bibliothèque nationale, rue de Richelieu », Louis Visconti, 1791-1853, Françoise Hamon (dir.), Paris, D.A.A.V.P., 1991, 268 p., p. 112-124.

43. Ibid., p. 117.

44. Voir Léon de Laborde, De l'organisation des bibliothèques dans Paris, $2^{e}$ lettre: Examen critique des projets présentés pour le déplacement de la Bibliothèque royale, Paris, A. Franck, 1845, 56 p. et aussi Françoise Hamon, "La Bibliothèque indésirable. Un feuilleton à rebondissements » ainsi que "Les Projets de Visconti », Louis Visconti, 1791-1853, op. cit., p. 125-132 et 133-141.

45. Voir Pierre Saddy, Henri Labrouste architecte, 1801-1875, Paris, C.N.M.H.S., 1977, 96 p. et Jean-Michel Leniaud (dir.), Des palais pour les livres, Labrouste, Sainte-Geneviève et les bibliothèques, Paris, éd. Maisonneuve \& Larose, 2002, 189 p.

46. Pascal répond ici à une lettre d'un certain L. Lambeaux qui lui demande si Labrouste a réutilisé la grille qui entourait la place Royale pour réaliser celle longeant la rue Vivienne.

47. Il s'agit de l'ancienne galerie du Trésor qui abritait en 1850 des collections ethnographiques.

48. L'hôtel Tubeuf.

49. Arch. adm. B.n.F., 2011/001/10021, lettre de Pascal à L. Lambeaux, secrétaire de la Commission du vieux Paris, 15 janvier 1906.

50. Roger-Armand Weigert, "Du palais Mazarin à la Bibliothèque nationale ", Mazarin, homme d'État et collectionneur, 1602-1661, exposition de la Bibliothèque nationale, Paris, 1961, p. XIII-XXX.

51. Né à Viterbe, élève du Dominiquin et de Pierre de Cortone. Voici ce qu'en dit le comte Léon de Laborde dans : De l'organisation des bibliothèques dans Paris, $4^{e}$ lettre : le palais Mazarin et les habitations de ville et de campagne au XVII siècle, op. cit., p. 11 : " C'est encore avec le Guide qu'il a le plus de ressemblance, tant par la légèreté de son coloris que par la grâce facile de son dessin. Il débuta dans la peinture à fresques au palais Barberini [...] Aujourd'hui, les villes d'Italie montrent ses fresques avec orgueil [...] sa Descente de Croix à Saint-Amboise, son Saint-Laurent à Viterbe, et dans l'église de Saint-Pierre de Rome, la mosaïque de sa Présentation, dont l'original est à la Chartreuse. »

52. Pour le détail de l'emplacement de ces peintures sur la voûte et leurs titres, voir Léon de Laborde, De l'organisation des bibliothèques dans Paris, 4 e lettre, op. cit., p. 13.

53. « Grimaldi jouissait à cette époque d'une grande réputation. Il était recherché par les plus riches seigneurs et renommé comme un des beaux talents de l'Italie. » Léon de 
Laborde, De l'organisation des bibliothèques dans Paris, $4^{e}$ lettre: le palais Mazarin et les habitations de ville et de campagne au XVII ${ }^{e}$ siècle, op. cit., p. 10. Grimaldi avait aussi œuvré dans la galerie basse du cardinal réservée à ses grands monuments de sculpture. Ces peintures existaient encore en 1754 : «elles avaient traversé tous les changements de destination subis par le local, qui conservait à cette époque les statues les plus mutilées. » Ibid., n. 46 p. 173. Elles furent badigeonnées par la suite.

54. Ibid., $\mathrm{n}^{\circ} 47$ p. 173.

55. « Bibliothèques », La Presse, 29 juillet 1863, B.n.F., dp. Mss, N.a.fr., 13026, f. 75.

56. Arch. adm. B.n.F., 2011/001/10003, lettre de Labrouste au ministre, 4 décembre 1867.

57. Ibid., lettre de Félix Perin à Labrouste, 16 décembre 1869.

58. Arch. adm. B.n.F., C 213, Lettre de Labrouste au ministre, 9 février 1869.

59. Ce ne sera pas le cas puisque lorsqu'André Chatelin, architecte de la bibliothèque nationale (1955-1981), découvre en 1975 des vrillettes dans les cerces de la voûte de la galerie Mazarine et qu'il les dégage en vue des travaux confortatifs nécessaires à l'installation de l'atelier de restauration, l'étude des fresques de la voûte et notamment de la partie centrale montre que les géants habillés par Romanelli à l'origine (dans la fresque Jupiter foudroie les géants) ont été dénudés par le restaurateur du XIX ${ }^{\mathrm{e}}$ siècle qui les a pourvus d'une musculature avantageuse (ill. 9 et 10).

60. Interrompue par les évènements de 1870-1871, la restauration dure jusqu'en 1872.

61. Arch. adm. B.n.F., 2011/001/10003, attachement $n^{\circ} 1$ de travaux autorisés du 19 au 23 avril 1869, d'Alexandre Denuelle, 9 juin 1869.

62. Arch. adm. B.n.F., 2011/001/10003, lettre de Perin à Labrouste, 16.12.1869. En effet sous Visconti, la galerie Mazarine servait de dépôt des manuscrits, la salle de lecture étant placée à l'extrémité sud de la galerie.

63. La salle du premier étage renfermait en effet le modèle en plâtre du Voltaire assis de Houdon exposé au Théâtre Français, modèle donné en 1810 à la Bibliothèque, pour la remercier de son hospitalité, par Mme Duvivier, au nom de Houdon. Son socle contenait, dans un coffret en métal doré, le cœur de l'écrivain. Celui-ci fut remis, en novembre 1864, au nom de la famille de Villette, par Léon Duval à Victor Duruy, qui le dépose à la Bibliothèque nationale.

La salle contenait aussi les médailles frappées en l'honneur de Voltaire, les correspondances manuscrites et les œuvres imprimées de l'écrivain.

B.n.F., Mss, N.a.fr. 13027. Papiers Henri Omont ; Deux articles : l'un du Petit Temps, 13 janvier 1898. L'autre intitulé :» À la Bibliothèque nationale. M. Roland-Marcel retrouve le cœur de Voltaire. Mais était-il perdu ?», 1924.

64. Jean-François Foucaud, «Extensions et travaux de la Bibliothèque nationale », op. cit., p. 346.

65. La salle contenait exactement 414 places : 344 places assises et 70 places debout.

66. Léon Labrouste, La Bibliothèque nationale, son début et ses accroissements, ses bâtiments et ses constructions, ses agrandissements, ses travaux, op. cit., p. 50-79. C. Imbert, « Les travaux d'agrandissement de la Bibliothèque nationale ", L'Architecture, 1933, p. 9-16. A. Goissaud, «Les agrandissements de la Bibliothèque nationale », La Construction moderne, 1937-1938, p. 114-120.

67. Le Siècle, 6 février et 8 juin 1864, B.n.F., dp. Mss, N.a.fr., 13026, f. 106 et 121. 
68. « Nous avons un service des eaux bien organisé dans la partie neuve voisine de la cour de la place Louvois, les pavillons contenant chacun un réservoir pour le service des pompiers ». Arch. adm. B.n.F., 2011/001/10005, lettre de Pascal à Barthélémy Saint Hilaire, 24 avril 1878.

69. Charles-Marie Widor, Notice sur la vie et les travaux de M. Jean-Louis Pascal, 4 juin 1837 - 16 mai 1920, op. cit., p. 8.

70. Ce n'est pas encore l'entrée dans l'axe de la place Louvois, puisqu'en 1886, Pascal écrit au ministre de l'instruction publique et des beaux-arts: " Monsieur, les travaux du bâtiment en façade sur la grande cour d'honneur de la Bibliothèque nationale sont à peu près terminés. Toutefois il reste encore à exécuter certains travaux urgents : la porte d'entrée de la Bibliothèque sur la place Louvois [...] La vraie entrée de la Bibliothèque nationale doit être dans l'axe de la place Louvois, par la grande porte encore fermée par une barrière en planches ». Arch. nat., $\mathrm{F}^{21}$ 2995, lettres du 8 juin et 12 juillet 1886.

71. Léon Labrouste, »Bibliothèque nationale, rue de Richelieu, à Paris, par M. H. Labrouste, membre de l'Institut. ", R.G.A.T.P., $\mathrm{n}^{\circ}$ XXXV, 1878, p. 144-152.

72. Arch. adm. B.n.F., 2011/001/10005, grands travaux, lettre de Pascal au ministre des travaux publics, février 1878.

73. Arch. nat., $\mathrm{F}^{21} 5854$.

74. «J'achève en ce moment, nous écrit M. Pascal, l'installation de casiers destinés à contenir la réserve des imprimés de la Bibliothèque, c'est-à-dire la collection des objets les plus remarquables de l'établissement, au point de vue de la rareté, de la perfection typographique, de la beauté des reliures. Cette installation définitive occupera tout le premier étage du nouveau bâtiment sur la place Louvois. Quand elle sera faite, on pourra agrandir le cabinet des médailles, campé provisoirement dans une partie des bâtiments sur la rue Richelieu et qui est tout à fait insuffisant ». «Chronique », R.G.A.T.P., $\mathrm{n}^{\circ} \mathrm{XXXIV}$, 1877, p. 81-85.

75. Actuel magasin des manuscrits orientaux.

76. Pour diminuer le bruit dans la grande salle publique de lecture, on pose en 1879 un linoleum venant de Linoleum Manufacturing Company, 21 boulevard Haussmann. Il s'agit d'un tapis de 0,006 m. d'épaisseur; on fera de même dans la salle de lecture de la rue Colbert puis plus tard à la salle de travail des manuscrits. Arch. adm. B.n.F., 2011/001/10006, grands travaux, 1880, lettre de Pascal au ministre des travaux publics, 21 mars 1880 .

77. Installée en bordure de la rue de Richelieu dans les nouveaux bâtiments construits par Labrouste le long de la nouvelle salle de lecture.

78. À établir à gauche de l'aile ouest de l'hôtel Tubeuf, dans la petite cour intérieure comprise entre le département des estampes et l'atelier de reliure, au-dessus du corps de bâtiment occupé par les cartes géographiques.

79. Ces maisons installées depuis le début du XVIII siècle à l'angle N.E. de la bibliothèque subsisteront jusqu'à l'expropriation de leurs propriétaires en 1881.

80. Il fallait réaliser un pavage simple, avec encadrement de granit, des grilles d'isolement pour les fenêtres, établir des fontaines, des appareils à gaz, et décorer la porte cochère.

81. Il le demandera jusqu'à son départ de la Bibliothèque, sans être entendu.

82. Arch. adm. B.n.F., 2011/001/10004, lettre de Pascal à Delisle, 12 juin 1876. 
83. Arch. nat., $\mathrm{F}^{21} 5854$, rapport de l'architecte de la bibliothèque nationale pour l'achèvement de la galerie Mazarine, J.-L. Pascal, 15 juin 1876. Le crédit demandé était de 200000 francs.

84. «Chronique », R.G.A.T.P., $\mathrm{n}^{\circ}$ XXXIV, 1877, p. 82.

85. Les vitrines, avec fermetures de précision, sont composées de montants, traverses, châssis contenant des glaces, tablettes et supports, tapisseries en velours et casiers. Elles sont en bois blanc, peint en noir avec filets dorés $(3,40 \mathrm{~m}$. de large, $0,60 \mathrm{~m}$. de profondeur, 3,15 m. de hauteur).

86. Arch. nat., $\mathrm{F}^{21} 5855$, lettre de Pascal au ministre des travaux publics, 28 décembre 1877.

87. Les trois tapisseries ont été affectées à la Bibliothèque nationale par arrêté ministériel du 9 novembre 1889.

88. Arch. adm. B.n.F., 2011/001/10005, grands travaux, 1879, lettre de Pascal au ministre des travaux publics, 29 janvier 1879.

89. Ces tapisseries figurent à l'Inventaire du mobilier de la B.N. sous les cotes : Inv. mob. 2. $\mathrm{n}^{\text {os }}$ 1347, 1348, 1349. Arch. adm. B.n.F., 2011/001/10006, lettre de Pascal à Mortreuil, 29 novembre 1889.

90. Peintre d'histoire né à Strasbourg, élève de Gleyre, entré à l'E.B.A. en 1857 ; il peint en 1877 Les Muses, plafond pour le palais de la Grande Chancellerie de la Légion d'honneur. Bellier de la Chavignerie, Louis Auvray, Dictionnaire général des artistes de l'École française, depuis l'origine des arts du dessin jusqu'à nos jours, architectes, peintres, sculpteurs, graveurs et lithographes, Paris, H. Loones, 1885, vol. 1, p. 512.

91. Pascal sera d'ailleurs nommé membre du jury chargé de juger le concours du prix des Gobelins de 1879.

92. La tapisserie est achevée le 15 février 1888.

Hauteur : 4,66 m., largeur : 7,67 m.

Prix : 76 151,25 fr.

Artistes-tapissiers : sujet : Justin Cochery, Camille Duruy, Thebaut

Bordure : Thebaut, Henri Cochery, Hocheid

93. La tapisserie est achevée le 14 septembre 1888.

Hauteur : 4,75 m., largeur : 2,73 m.

Prix : 32 558,98 fr.

Artistes-tapissiers : sujet : Michel, Gibier, Vacher.

Bordure : Perrot, Beauboeuf

94. La tapisserie est achevée le 9 novembre 1887

Hauteur : 4,72 m., largeur : 2,63 m.

Prix : 24 645,84 fr.

Artistes-tapissiers : sujet : Étienne Mary, Paul Marie

Bordure: Ralhoff, Plistat

Arch. adm. B.n.F., 2011/001/10006, désignation des tapisseries de haute lisse affectées à la Bibliothèque nationale, 23 novembre 1889.

95. Ces deux tapisseries : Le Manuscrit et L'Imprimé, ainsi que Les lettres, les Sciences et les Arts dans l'Antiquité, furent envoyées à l'exposition universelle de Chicago en 1893. Arch. 
nat., $\mathrm{F}^{21}$ 5863, lettre de Pascal au ministre de l'instruction publique et des beaux-arts, 19 janvier 1893.

96. Trophée pyramidal en bronze de 1,35 m. de haut sur $85 \mathrm{~cm}$. de large et porté par un soubassement. Henry Marcel, Henri Bouchot, Ernest Babelon, Paul Marchal, Camille Couderc, La Bibliothèque nationale, Paris, éd. H. Laurens, 1907, 129 p., p. 10.

97. L'entreprise Barbedienne est située 30 boulevard Poissonnière. Arch. adm. B.n.F., 2011/001/10005, grands travaux, 1878, lettre de Pascal, 30 janvier 1878.

98. Il s'y trouve toujours en 1926. Arch. nat., $F^{21}$ 5862, rapport de Defrasse, inspecteur général de la $4^{\mathrm{e}}$ division des bâtiments civils, au sous-secrétaire d'État aux beaux-arts, 10 avril 1926. Lorsqu'Ernest Recoura, successeur de Pascal à la Bibliothèque nationale à partir de 1912, rénove la galerie Mazarine et son vestibule en 1927, grâce au legs dû à Florence Blumenthal, il démonte le Parnasse français. L'antichambre de la galerie Mazarine est alors appelée « salle Blumenthal ».

99. Henry Marcel, Henri Bouchot, Ernest Babelon, Paul Marchal, Camille Couderc, La Bibliothèque nationale, op. cit., p. 10.

100. Le comité consultatif s'est prononcé sur les places à donner dans la galerie Mazarine. Charles V, au milieu de la galerie.

À sa gauche : François 1er, Peiresc, Marolles, Budé, Van Praët, (les deux derniers occupant les deux côtés de la porte d'entrée de la galerie).

À sa droite: Louis XIV, Colbert, Mabillon, Caylus, Dupuy, de Thou, (Dupuy et de Thou seront placés aux deux côtés de la porte d'entrée donnant sur le département des manuscrits).

Arch. adm. B.n.F., 2011/001/10006, lettre de Théodore Mortreuil à Pascal, 10 février 1882.

101. Douze bustes $(0,80 \mathrm{~m}$. de haut y compris les piédouches de $0,12 \mathrm{~m}$.) sont commandés pour remplir les niches de la galerie Mazarine. Arch. adm. B.n.F., 2011/001/10005, grands travaux, 1879, lettre de Pascal au sous-secrétaire d'État, 27 février 1879.

102. Originaire d'Aix-en-Provence, Peiresc se lie en 1597 avec Pierre-Antoine de Rascas de Bagarris, intendant des médailles et antiques d'Henri IV, qui l'initie à la numismatique. Docteur en droit, astronome, historien, archéologue, amateur d'art, il réunit une collection de 18000 médailles entrée au cabinet du Roi Louis XIV.

103. L'abbé de Marolles, érudit, collectionneur, écrivain d'art, titulaire d'un monastère en Touraine, vendit à Louis XIV en 1667 sa collection de 123000 pièces dessinées ou gravées pour 30400 livres. Il fut le premier conservateur du cabinet des estampes du roi, aménagé dans une des chambres de l'hôtel Colbert, rue Vivienne.

104. Conservateur du département des imprimés de 1784 à 1837.

105. Pierre et Jacques Dupuy sont nommés gardes de la Librairie du roi en 1635, à la suite de Nicolas Rigault, dont ils refondent le catalogue, premier travail d'ensemble auquel aient donné lieu les livres imprimés et manuscrits de la Bibliothèque du roi. À la mort de Jacques survenue en 1656, la riche collection de livres imprimés des deux frères est léguée à Louis XIV ; elle constitue le véritable noyau du département des imprimés, plus de 9000 volumes, presque tous reliés aux armes de la famille Dupuy.

106. Fils de Christophe de Thou, premier président du parlement de Paris, il fut magistrat, historien, écrivain. Il fut nommé grand maître de la Librairie du roi Henri IV en 1593. 
107. Le legs du comte de Caylus, antiquaire, fit entrer dans la collection royale en 1765 des objets égyptiens, des bronzes, 72 vases peints. Dans son célèbre Recueil d'antiquités, il a publié et commenté son riche musée.

108. Sculpteur parisien, élève de Granfils et Jouffroy, il entre à l'E.B.A. en 1853 et remporte le $1^{\text {er }}$ grand prix de Rome en 1863 ; il réalise beaucoup de portraits en buste, deux statues de marbre pour le jardin du Luxembourg (Narcisse, 1869 et Arion, 1870), un Saint Jean de Matha en plâtre pour l'église Sainte-Geneviève (1876). Émile Bellier de la Chavignerie, Louis Auvray, Dictionnaire général des artistes de l'École française, op. cit., vol. 1, p. 770 .

109. Sculpteur, élève de Jouffroy, entré à l'E.B.A. en 1858 qui réalise beaucoup de portraits en buste et médaillons. Ibid, vol. 1, p. 150.

110. Hubert-Noël Louis, dit Louis Noël, né à Saint-Omer dans le Pas-de-Calais, élève de Jouffroy. Ibid., p. 1061.

111. Sculpteur et peintre parisien, élève de Maillet, Duret et Cavelier, il est $1^{\mathrm{er}}$ grand prix de Rome en 1870 et a réalisé la Loi et la Prudence, statues de pierre pour la façade du pavillon de Flore aux Tuileries, ainsi que de nombreux portraits en buste. Ibid., vol. 1, p. 874-875.

112. Sculpteur né à Toulouse, élève de Jouffroy et Falguière, $1^{\text {er }}$ grand prix de Rome en 1871, il sculpte Jacob et l'ange, bas-relief de plâtre (1874), Persée et la Gorgonne, groupe en plâtre de 1876, Vélléda, statue de marbre (1877), exposée à l'exposition universelle de 1878. Ibid., vol. 2, p. 36.

113. Arch. adm. B.n.F., 2011/001/10005, grands travaux, 1879, lettre de Pascal au soussecrétaire d'État, 10 mars 1879.

114. Sculpteur animalier, né à Paris, élève de Rude qui a remporté de nombreuses médailles ; il remplace Antoine Barye comme professeur au Museum. On lui doit aussi de nombreuses statues équestres dont la Jeanne d'Arc de la place des Pyramides à Paris (1880) ou encore Louis d'Orléans, frère de Charles VI, pour le château de Pierrefonds (1870). Statuaire officiel de la III é République, il rentre à l'Institut en 1892. Émile Bellier de la Chavignerie, Louis Auvray, Dictionnaire général des artistes de l'École française, op. cit., vol. 1, p. 588.

115. Sculpteur né à Béziers (Hérault), élève de Dumont, qui remporte le $1^{\mathrm{er}}$ grand prix de Rome en 1874. Il sculpte Pierre Lescot et Germain Pilon, pour l'Hôtel de Ville de Paris. Ibid., p. 803.

116. Arch. adm. B.n.F., 2011/001/10006, grands travaux, 1880, lettre du sous-secrétaire d'État aux beaux-arts à Pascal, 30 janvier 1880. Le prix sera de 3400 francs.

117. Domicilié au n ${ }^{\circ} 99$ de la rue de Vaugirard à Paris.

118. Sculpteur né à Aix (Bouches-du-Rhône), élève de Dumont. Émile Bellier de la Chavignerie, Louis Auvray, Dictionnaire général des artistes de l'École française, op. cit., vol. 1, p. 791.

119. Arch. adm. B.n.F., 2011/001/10006, lettre du directeur général des beaux-arts Mantz à Pascal, $1^{\text {er }}$ août 1882 .

Honoré Icard est un sculpteur né à Tourtouze dans l'Ariège, élève à l'E.B.A. de A. Dumont et A. Muller. Deux de ses statues (plâtre) seront exposées à l'exposition universelle de 1878: Saint Jérôme (1876) et David devant Saül. Émile Bellier de la Chavignerie, Louis Auvray, Dictionnaire général des artistes de l'École française, op. cit., vol. 1, p. 801. 
120. Arch. adm. B.n.F., 2011/001/10006, lettre du $1^{\mathrm{er}}$ septembre 1882 de Mantz à Pascal.

121. Ibid., lettre du directeur des beaux-arts, A. Kempfer à Pascal, 13 mai 1886.

122. Le coût est de 20000 francs. Arch. nat., $F^{21}$ 5857, lettre de Pascal au ministre de l'instruction publique et des beaux-arts, 30 août 1882 .

123. Sculpteur né à Castres (Tarn), élève de Jouffroy, il entre à l'E.B.A. en 1853. Il réalise des bustes en marbre du Général Auger (1864) et d'Alfred de Vigny (1867) pour les galeries de Versailles. En 1865, il sculpte la Cigale, statue de marbre réexposée au Champ de Mars en 1867. Émile Bellier de la Chavignerie, Louis Auvray, Dictionnaire général des artistes de l'École française, op. cit., vol. 1, p. 193.

124. Ce que faisait depuis longtemps le British Museum. Arch. nat., $\mathrm{F}^{21} 5855$, rapport de J.L. Pascal au ministre de l'instruction publique et des beaux-arts, sur les travaux à exécuter à la bibliothèque nationale., mai 1876, rapport qui complète un rapport adressé en octobre 1875 à l'Administration relativement à l'ensemble des travaux à exécuter à la Bibliothèque nationale.

125. C'est le $n^{\circ} 40$ sur le plan édité en 1878 (ill. 11).

126. Situé dans l'aile ouest de l'hôtel Tubeuf, jouxtant la galerie Mazarine.

127. L'atelier de la reliure situé dans l'aile ouest de l'hôtel Tubeuf (ill. 15).

128. Arch. adm. B.n.F., 2011/001/10005, devis descriptif de Pascal, 27 décembre 1878, pour la construction d'un atelier photographique à la Bibliothèque nationale.

129. Arch. nat., $F^{21} 750$, B.N., entretien 1877, chapitre 21, lettre de Pascal au ministre des travaux publics, 26 décembre 1876. La réserve n'est pas encore installée au $1^{\mathrm{er}}$ étage de l'aile donnant sur le square Louvois.

130. Galerie reliant le palier situé entre l'extrémité nord de la galerie Mazarine et le $1^{\mathrm{er}}$ étage du bâtiment est de la cour d'honneur, et les bâtiments le long de la rue de Richelieu. Cette galerie de bois longeait la nouvelle salle de lecture d'Henri Labrouste.

131. Arch. adm. B.n.F., 2011/001/10005, grands travaux, 1878, lettre de Léopold Delisle à Pascal, 11 février 1878.

132. Le budget se décompose ainsi: - Bâtiments en façade sur la place Louvois. Installation au $1^{\mathrm{er}}$ étage de la réserve des imprimés : $60000 \mathrm{fr}$; agrandissement du cabinet des médailles et transfert de la réserve des imprimés : $50000 \mathrm{fr}$; installation d'un cabinet de photographie : $40000 \mathrm{fr}$; commencement des travaux de construction des anciens bâtiments sur la rue Colbert : 172000 fr. Soit un total de 322000 fr. Arch. nat., $F^{21}$ 5855, rapport du maître des requêtes A. Tréteau au ministre des travaux publics, 24 mars 1877, d'après le rapport de Pascal.

133. Jean Formigé, Notice sur la vie et les travaux de M. J.-L. Pascal, 4 juin 1837-16 mai 1920, Institut de France, Académie des beaux-arts, 12 novembre 1921, 12 p., p. 8.

134. Charles-Marie Widor, Notice sur la vie et les travaux de M. Jean-Louis Pascal, 4 juin 1837-16 mai 1920 , op. cit., p. 8.

135. Léon Labrouste, "Bibliothèque nationale, rue de Richelieu, à Paris, par M. H. Labrouste, membre de l'Institut », op. cit., p. 144-152. 


\section{RÉSUMÉS}

Fondée par Charles $\mathrm{V}$ dans la seconde moitié du XIV ${ }^{\mathrm{e}}$ siècle, au Louvre, la première Bibliothèque royale est vendue. On situe donc les vrais débuts de la Bibliothèque du roi au temps de Louis XI dont les collections ne sont pas dispersées après la mort. Charles VIII, Louis XII et François $1^{\mathrm{er}}$ l'installent à Amboise, à Blois et à Fontainebleau. C'est Charles IX qui ramène la Bibliothèque à Paris en 1567 et, en 1666, Colbert l'installe dans son hôtel de la rue Vivienne, au bout de ses jardins, dans deux maisons particulières. La faillite de la banque de John Law, qui avait investi le palais de Mazarin situé de l'autre côté de la rue Vivienne, en face de la maison de Colbert, donne l'idée au Régent Philippe d'Orléans de lui consacrer ces bâtiments désertés par le financier. C'est en 1721 que la Bibliothèque royale s'installe dans le quadrilatère Richelieu. Lorsqu'Henri Labrouste devient architecte de la Bibliothèque en 1854, succédant à Louis Visconti, il restaure l'hôtel Tubeuf, noyau initial du palais Mazarin, mais détruit les bâtiments construits à la suite, héritage des XVII et XVIII ${ }^{\mathrm{e}}$ siècles. Jean-Louis Pascal, lorsqu'il succède à Henri Labrouste en 1875, inaugure une politique nouvelle, celle du respect pour les bâtiments hérités des siècles passés. Ses premiers travaux se font d'abord dans le prolongement de ce qu'avait initié Labrouste: il parachève les bâtiments construits par ce dernier le long de la rue de Richelieu et y installe, en face du square Louvois, la réserve des imprimés. Il transforme ensuite la galerie Mansart en salle d'exposition et crée un atelier de photographie.

Founded by Charles the Fifth in the second part of the XIV ${ }^{\text {th }}$ century at the Louvre, the first National Library was sold. So Louis the $\mathrm{XI}^{\text {th }}$ 's reign, whose the collections were not split up after his death, is usually considered as the reign of the real beginnings of the Royal Library. Then the Library is established in Amboise, Blois and Fontainebleau by Charles the VIII ${ }^{\text {th }}$ Louis the XII ${ }^{\text {th }}$ and François the First. Charles the IX ${ }^{\text {th }}$ takes the Library back in 1567, and in 1666, Colbert settles it in his private mansion Rue Vivienne, behind his gardens, in two houses. John Law bank's bankruptcy let the Régent Philippe d'Orléans think about the Palais Mazarin : just in front of Colbert's home, on the other side of the Rue Vivienne and previously occupied by John Law, these empty buildings could be used for the Library too. The royal Library takes place here in 1721 . When Henri Labrouste became the official architect of the Library in 1854, after Louis Visconti, he restores the Hôtel Tubeuf, the first core of the Palais Mazarin but destroys the buildings built in the XVII ${ }^{\text {th }}$ and XVIII ${ }^{\text {th }}$ century. When Jean-Louis Pascal succeeds Henri Labrouste in 1875, he opens a new era, where buildings from former centuries are respected. His first works are firstly the prolongation of what have been initiated by Labrouste : he completes his buildings along the Rue de Richelieu and sets, in front of the Louvois Square, the Printed Documents Storage Room. Then he transforms the Galerie Mansart into a venue and establishes a photography studio.

Die von Karl V. in der zweiten Hälfte des 14. Jahrhunderts im Louvre begründete königliche Bibliothek ist in späteren Jahren wieder verkauft und zerstreut worden. Der wirkliche Beginn einer königlichen Bibliothek wird daher zu Zeiten Ludwigs XI. vermutet, dessen Sammlungen nach seinem Tod nicht veräußert werden. Karl VII., Ludwig XII. und Franz I. richten jeweils Bibliotheken in Amboise, Blois und Fontainebleau ein. Erst Karl IX. führt die Sammlungen 1567 wieder nach Paris zurück, wo Colbert die Bestände 1666 in seinem Hôtel particulier in der Rue Vivienne in zwei Häusern am Ende der Gärten unterbringt. Im Zuge des Konkurses der Bank von 
John Law, der den dem Anwesen von Mazarin gegenüberliegenden Palast erworben hatte, schlägt der Regent Philipp von Orléans vor, die verlassenen Gebäude für die Bibliothek zu nutzen. 1721 wird die königliche Bibliothek auf die Parzelle an der Rue Richelieu verlegt. Als Henri Labrouste 1854 von Louis Visconti das Amt des Architecte de la Bibliothèque übernimmt, setzt er das Hôtel Tubeuf, den ursprünglichen Kern des Palais Mazarin, wieder instand, lässt jedoch die im 17. und 18. Jahrhundert im Umfeld errichteten Gebäude abbrechen. Als Jean-Louis Pascal 1875 die Nachfolge von Labrouste antritt, schlägt er eine neue Leitlinie ein, die sich größerem Respekt vor den bestehenden Bauten verpflichtet zeigt. Zunächst setzt er die von Labrouste entworfenen Bauvorhaben mit der Vollendung der entlang der Rue Richelieu geplanten Flügel fort und richtet gegenüber des Square Louvois die Abteilung für Drucke ein. Anschließend verwandelt er die Galerie Mansart in einen Ausstellungssaal und begründet ein Atelier für Fotografie

\section{INDEX}

Index chronologique : époque contemporaine

Mots-clés : restauration, bibliothèque

Schlüsselwörter : Restaurierung, Bibliothek

Keywords : restitution, library

\section{AUTEUR}

\section{ANNE RICHARD-BAZIRE}

Anne Richard-Bazire est docteur de l'École pratique des hautes études (section des sciences historiques et philosophiques), diplômée en histoire de l'art contemporain et en archéologie égyptienne. Elle est actuellement chargée du cours de synthèse de l'Histoire de l'architecture occidentale, $\mathrm{XIX}^{\mathrm{e}}-\mathrm{XX}$ siècles, à l'École du Louvre. Elle a rédigé différents articles : « L'habitat néo-gothique à Paris au XIX ${ }^{\mathrm{e}}$ siècle ", La demeure médiévale à Paris, Étienne Hamon et Valentine Weiss (dir.), Paris, 2012, Archives Nationales, p. 250-257 ; « La salle Ovale ou l'histoire des dysfonctionnements dans les chantiers parisiens du XIX ${ }^{\mathrm{e}}$ siècle à travers l'exemple de la Bibliothèque nationale ", La Collégialité et les dysfonctionnements dans la décision administrative, JeanMichel Leniaud et François Monnier (dir.), Paris, 2011, École Pratique des Hautes Études, p. 103-112 ; «Jean-Louis Pascal ou le respect du patrimoine », article mis en ligne sur le site "Archicab", le 4 décembre 2011, http://www.jeanfrancoiscabestan.com/bnfrichelieu.html ; « Le concours pour la reconstruction de l'Hôtel de Ville de Paris (1872-1873), un échec pour l'architecte Jean-Louis Pascal », Livraisons d'histoire de l'architecture, $\mathrm{n}^{\circ} 19,2010$, p.111-134 ; « L'escalier d'honneur de la Bibliothèque nationale de la rue de Richelieu », Sites et Monuments, $\mathrm{n}^{\circ}$ 207, oct.-nov.-déc. 2009, p. 22-24 ; « La salle Ovale », Revue de la Bibliothèque nationale de France, $\mathrm{n}^{\circ}$ 30, 2008, 96 p., p. 32-39 ; « La faculté de médecine et de pharmacie de Bordeaux par Jean-Louis Pascal (1876-1888 et 1902-1922) », Livraisons d'histoire de l'architecture, $\mathrm{n}^{\circ}$ 13, 2007, p.105-120 ; « Le ministère de l'agriculture de la rue de Varenne par Emmanuel Brune, l'alliance de la science et de l'art », Livraisons d'histoire de l'architecture, $\mathrm{n}^{\circ}$ 8, 2004, p. 81-94; « Le parvis de Notre-Dame » et « Le square Jean XXIII ", Autour de Notre-Dame, sous la dir. de Alain Erlande-Brandeburg, Jean-Michel Leniaud, François, Christian Michel, Paris, D.A.A.V.P., 2003, 269 p., p. 240-242 et 243-245 ; « Les réalisations de Pascal », et «L'École des beaux-arts » avec Fabienne Doulat, Les bibliothèques parisiennes, architecture et décor, sous la dir. de Myriam Bacha et Christian Hottin, Paris, D.A.A.V.P., 2002, 272 p., p. 115-117 et 132-134 ; « Un siècle de réflexion sur la construction des bibliothèques ", Des palais pour les livres, Labrouste, Sainte-Geneviève et les bibliothèques, sous la dir. de Jean-Michel 
Leniaud, Paris, éd. Maisonneuve \& Larose, 2002, 189 p., p. 58-69 ; « Jean-Louis Pascal et la création de la salle des périodiques de la Bibliothèque nationale (1883-1936) », Livraisons d'histoire de l'architecture, $\mathrm{n}^{\circ}$ 1, 2001, p. 105-125. Elle a dirigé avec Martin Drouin : La Sélection patrimoniale, Québec, Éditions MultiMondes, coll. Cahiers de l'Institut du patrimoine de l'UQAM, nº 13, 2011, 388 p. Elle a participé en octobre 2001 au colloque international organisé par la Bibliothèque Sainte-Geneviève à Paris : « Henri Labrouste et la Bibliothèque Sainte-Geneviève, Histoire et actualité », en octobre 2009 au colloque : «Victor Laloux, un architecte dans sa ville » organisé à Tours par le Conseil général d'Indre-et-Loire, en juillet 2010 aux journées d'études organisées par le Centre-Ledoux-Université Paris I, la Commission du Vieux Paris et l'Association pour la Sauvegarde et la Mise en valeur du Paris Historique, sur le thème : «BNF Richelieu : un projet en questions »; en octobre 2010, elle a été la coordinatrice scientifique avec Martin Drouin de la Sixième Rencontre internationale des jeunes chercheurs en patrimoine sur le thème de la « Sélection patrimoniale », organisée conjointement par l'Université du Québec à Montréal et l'École des Chartes à Paris, sous la direction de Luc Noppen, Lucie K. Morisset (UQAM) et JeanMichel Leniaud (École des chartes). Elle a participé en janvier 2011 aux Journées annuelles de l'équipe Histoire du droit public et de l'administration, EPHE, sous la direction de François Monnier, directeur de l'UR, Jean-Michel Leniaud et Jean-Miguel Pire sur le thème : « Les dysfonctionnements dans la décision administrative », en novembre 2011 à la journée d'études de l'équipe Histara de l'EPHE, organisée à l'INHA par Jean-François Belhoste, directeur d'études à l'EPHE, et Isabelle Parizet, maitre de conférences à l'EPHE, sur le thème : « Architectures bancaires : entre le Beau et l'Utile ", en décembre 2012 au colloque international organisé par l'INHA, Le musée d'Orsay et la Maison des centraliens : « William Le Baron Jenney (1832-1907). De l'École centrale de Paris aux gratte-ciel de Chicago ». Elle a réalisé de mars 2012 à janvier 2013 pour le compte de l'OPPIC les recherches historiques préalables à la restauration des galeries Mansart, Mazarine et de la « chambre de Mazarin » à la Bibliothèque nationale Richelieu sous la direction de l'architecte en chef des monuments historiques A. de Saint-Jouan. Adresse électronique : anne.richardbazire@hotmail.com. 